\title{
Bio-Inspired Canopies for the Reduction of Roughness Noise
}

\author{
Ian A. Clark ${ }^{\mathrm{a}}$
}

(C) 2016. This manuscript version is made available under the Elsevier user license http://www.elsevier.com/open-access/userlicense/1.0/ 
Virginia Polytechnic Institute and State University, Blacksburg VA 24061, USA

\author{
Conor A. Daly \\ University of Cambridge, Cambridge CB3 OWA, UK \\ William Devenport and W. Nathan Alexander \\ Virginia Polytechnic Institute and State University, Blacksburg VA 24061, USA \\ Nigel Peake \\ University of Cambridge, Cambridge CB3 OWA, UK \\ Justin W. Jaworski \\ Lehigh University, Bethlehem PA 18015, USA \\ Stewart Glegg \\ Florida Atlantic University, Boca Raton FL 33431, USA
}

\begin{abstract}
This work takes inspiration from the structure of the down covering the flight feathers of larger species of owls, which contributes to their ability to fly almost silently at frequencies above $1.6 \mathrm{kHz}$. Microscope photographs of the down show that it consists of hairs that form a structure similar to that of a forest. The hairs initially rise almost perpendicular to the feather surface but then bend over in the flow direction to form a canopy with an open area ratio of about $70 \%$. Experiments have been performed to examine the noise radiated by a large open area ratio canopy suspended above a surface. The canopy is found to dramatically reduce pressure fluctuations on the underlying surface. While the canopy can produce its own sound, particularly at high frequencies, the reduction in surface pressure fluctuations can reduce the noise scattered from an underlying rough surface at lower frequencies. A theoretical model is developed which characterizes the mechanism of surface pressure reduction as a result of the mixing layer instability of flow over forest canopies.
\end{abstract}

Keywords: Roughness Noise, Bio-Inspired, Noise Reduction

\title{
1. Introduction
}

Many species of owl are able to hunt in effective silence, a feat which is believed to be linked to three unique physical attributes ${ }^{1}$ : a comb of evenly-spaced bristles along the wing leading-edge; a compliant and porous fringe of feathers at the trailing-edge; and a velvety down material distributed over the upper wing surface. Early work by Kroeger et al. ${ }^{2}$ investigated these features experimentally with live owls, where the removal of the leading-edge comb resulted in increased aerodynamic noise as well as a loss of flight control authority. Related experiments by Hersh et al. ${ }^{3}$ for airfoils with leading-edge serrations demonstrated their ability to reduce aerodynamic noise. Trailing-edge noise is a known major contributor to airframe noise, and recent theoretical modeling by Jaworski and Peake ${ }^{4,5}$ has suggested that the porous 
and compliant nature of the trailing edge can effectively eliminate the edge scattering mechanism. Specifically, they show, in a scaling sense, that trailing-edge noise levels may be reduced to levels comparable to ordinarily secondary noise mechanisms such as roughness noise.

Work on the downy surface, particularly as it relates to the acoustics of owl flight, is somewhat limited. Lilley ${ }^{6}$ postulated by process of elimination that the downy material, which creates a rough but compliant upper wing surface, contains a mechanism to diminish aerodynamic noise at the source for a broad frequency range relevant to the owl. Bachmann et al. ${ }^{7}$ measured the barbules (downy fibers) of barn owls and compared them in length and frequency density to pigeon feathers. In their discussion of the morphology of the barbules, the impact of these barbules on the noise generation is left an open question but they speculate that they reduce the noise of rubbing between feathers and perhaps alter the boundary layer. Additional details of the feather anatomy, including that of the velvety surface, are given later by Bachmann $^{8}$. A similar feather comparison (this time between an eagle owl and a buzzard) by Chen et al. ${ }^{9}$ arrives at essentially the same conclusions, noting that the length of the barbules creates a porous structure that could affect the acoustics. However, like the Bachmann et al. ${ }^{7}$ study, the function of the barbules/down is not clarified, and in the case of Chen et al. ${ }^{9}$, it is speculated that it behaves as an ordinary sound absorber for high frequencies. Later investigations by Klan et al. ${ }^{10,11}$ installed artificial hairy surfaces to match the measured length and density of the barn owl barbules in the study of Bachmann et al. ${ }^{7}$. The focus was on the effect of the hairs on the fluid flow and not on noise generation/suppression. However, there is no effort here or elsewhere to measure the elastic properties of the barbules or try to match them quantitatively to an elastic barbule material in their experiments.

The present work seeks to examine the hypothesis of Lilley ${ }^{6}$ with a study of the owl down and subsequent theoretical modeling and aeroacoustic experiments informed by the structure of the down. The organization of this paper is as follows. First, a photographic study is described that is carried out with feathers of well-known silent owl species, which identifies a 'forest' structure that creates a buffer between the turbulent boundary layer flow and the wing (or wall) surface. This concept is then explored experimentally for specialized smooth and rough surfaces veiled by various porous materials to emulate the effect of the owl down. This work is then investigated theoretically to describe the attenuation of surface pressure fluctuations with a mixing layer instability model. The paper is then concluded with a summary of findings and results.

\section{The Structure of the Owl Feather Down}

As discussed above, one possible noise-reducing mechanism of the owl's feathers is the downy coating consisting of flexible fibers on the surface of the feather. In order to investigate this further and to prepare for experimentation, a visual study of some owl feathers was performed to understand the structure of the fibers and to determine the average length and density of the fibers. 
Four primary feathers were studied which were molted by an eagle owl, a great gray owl, and a snowy owl, Figure 1. Multiple locations on one feather were studied to characterize changes in fiber size and density with position. The feathers were studied and imaged using a Nikon SMZ1500 microscope and a Nikon D300S Digital SLR camera. An Edmund 53713 micrometer was used to scale the images. Aside from scaling the 2D images, the focal adjustment of the microscope was calibrated in order to obtain measurements in the vertical plane.

In cross section (e.g. Figure 2) the feathers are seen to be covered in hairs that typically rise $1 \mathrm{~mm}$ off the surface. The samples for these pictures were approximately taken from the center span and chord of the feathers. In different parts of the owl's wing, the hairs rise at different angles to the feather surface. The individual hairs are also barbed, a feature that is particularly clearly seen in planform view (Figure $3 \mathrm{c})$. The hairs tend to be longer near the root of the feathers and shorter near the tip.

Using shallow depth of focus in planform view it is clear that the surface structure is organized into layers (Figure 3). The lowest layer (the substrate) is formed by an apparently impermeable mat of fibers (Figure 3a). Rising from these fibers is a 'forest' of hairs visible in Figure 3b. After rising about $0.5 \mathrm{~mm}$ off the substrate, the hairs bend over creating a 'canopy' (Figure 3c), which is enhanced by the barbs on the individual hairs.

Overall, the average vertical thickness of the downy coating is 0.25 to $1.5 \mathrm{~mm}$. The coating is thicker near the root of the feather and thinner near the tip of the feather. The average lengths of the fibers are on the order of 1-2 mm. Based on the number and arrangement of the fibers above the surface of the feather, it is estimated that the canopy formed by the down has an open area ratio of approximately $70 \%$, which is within the range of $45-90 \%$ given by Bachmann ${ }^{8}$ for those areas of feathers which are exposed to the flow. The fibers feature very short $(\sim 0.05 \mathrm{~mm})$ barbs along the lengths of the fibers which fill some of the area between the fibers.

The length scales of the owl's downy coating are very small, and the flow Reynolds number based on the length of the fibers is estimated to be around 100, while that based on the diameter of the fibers or the lengths of the barbs could be as low as 7. It was also evident that the fibers are oriented in a certain direction which would be aligned with the flow during normal flight.

\section{Experimental Apparatus and Instrumentation}

Experimental studies were performed on the aeroacoustics of flow surfaces with configurations inspired by the results of the photographic study. Surface pressure fluctuations and sound produced by a boundary layer flowing over smooth and rough surfaces were measured. The effects of placing a series of high open area ratio fabric coverings just above these surfaces was examined. The fabric acted as a canopy over the surface roughness in order to simulate the downy coating of the owl's feathers. The 
rough surfaces included two surfaces (hemispherical and sandpaper) whose roughness noise characteristics are well known from previous studies ${ }^{12,13}$.

\subsection{Wind Tunnel Facility}

The Virginia Tech Anechoic Wall-Jet Wind Tunnel was used, shown in Figure 4. This wind tunnel produces a quiet two-dimensional wall jet within an acoustic enclosure that flows and decays over a $3 \mathrm{~m}$ long smooth flat plate. The wall jet consists of a boundary layer flow adjacent to a flat plate topped by a mixing layer. This open upper boundary allows for the measurement of far-field noise without the need to place microphones in or near the flow. The small scale of the flow source needed for a wall jet allows background sound to be reduced to a minimum and the natural decay of this flow enables any edges (and potential scattering sources) to be positioned away from regions of significant flow velocity. The extremely low background noise levels allow for high signal-to-noise ratio, single microphone measurements of roughness noise over a range of carefully controlled boundary layer conditions, as well as measurements of supporting aerodynamic data.

The wall jet exhausts from a nozzle $1219 \mathrm{~mm}$ wide and $12.7 \mathrm{~mm}$ high with an initial velocity that was varied between 20 and $60 \mathrm{~m} / \mathrm{s}$. By the time the jet exits from under the baffle it has reached a fullydeveloped form with two-dimensional flow over its central $900 \mathrm{~mm}$ region. The scaling parameters of its mean velocity profile downstream of this point can be accurately described using the power-law relations of Wygnanski et al. ${ }^{14}$ :

$$
\begin{aligned}
& \frac{U_{m}}{U_{j}}=1.6814 R e_{j}^{n+1} R e_{x}^{n} \\
& \frac{\delta}{b}=0.1153 R e_{j}^{p-2} R e_{x}^{p}
\end{aligned}
$$

where $U_{m}$ is the maximum velocity in the mean velocity profile (at the boundary layer edge), $U_{j}$ is the jet exit velocity, $\delta$ is the boundary layer thickness (i.e. the distance from the wall to $U_{m}$ ), $b$ is the nozzle height, and $R e_{j}$ and $R e_{x}$ are jet-exit velocity Reynolds numbers based, respectively, on the nozzle height and distance $x$ downstream from the nozzle. The values $n$ and $p$ were found experimentally to be -0.4702 and 0.9099 , respectively. Flow properties implied by the above relations for the location $x=1410 \mathrm{~mm}$ (the streamwise center of the surfaces tested) are given in Table 1.

\subsection{Rough Surfaces}

Two rough surfaces were tested. Both a hemispherical roughness fetch and a sandpaper roughness fetch were positioned symmetrically with respect to the wall-jet plate centerline, were centered at $x=$ $1410 \mathrm{~mm}$, and were attached directly to the wall-jet plate. The hemispherical roughness, pictured in Figure 5a, extended $300 \mathrm{~mm}$ in the streamwise direction and $600 \mathrm{~mm}$ spanwise and consisted of $3 \mathrm{~mm}$ - 
radius hemispheres spaced $16.5 \mathrm{~mm}$ center to center in a square array. This surface was identical to that tested previously as part of roughness noise studies conducted by Alexander ${ }^{12}$. The second surface, pictured in Figure 5b, was formed by a sheet of 20-grit sandpaper roughness extending $200 \mathrm{~mm}$ streamwise and $600 \mathrm{~mm}$ spanwise. This type of surface, tested previously by Devenport et al. ${ }^{13}$, has a nominal grain size of $0.95 \mathrm{~mm}$, a grain density of 0.23 grains $/ \mathrm{mm}^{2}$, and an RMS roughness height of $0.206 \mathrm{~mm}$. The edges of these roughness patches were taped and faired to the surrounding wall using 0.12 mm thick aluminum tape. Devenport et al. ${ }^{13}$ demonstrated that the perimeter of the roughness substrate and the tape produces no detectable noise with this arrangement.

\subsection{Fabric Canopies}

Five mesh-like polyester or nylon fabrics, later down selected to four, were used to mimic the effect of the canopy portion of the owl down. The fabrics were selected based on qualitative similarity to the structure of the owl's downy coating (high open area ratio, interlocking fibers). Pictures of the fabrics are shown in Figure 6. Characteristics of the fabrics are listed in Table 2.

The fabrics are structured as meshes with a 2.5:1 ratio of pore sizes, a 5:1 ratio of thread diameters, and open area ratios from $38 \%$ to $76 \%$. In terms of filament size and open area ratio, fabric 5 most closely simulates the owl-down canopy, but even with this finest fabric the thread diameter is about three times the estimated diameter of the owl's hairs. The least similar is fabric $4-$ a much heavier mesh than the other fabrics. The fabric meshes are not isotropic surfaces, although some display rotational symmetries. All fabrics were tested with the vertical direction in Figure 6 aligned with the flow direction, thus placing a significant fraction of the fibers perpendicular to the flow direction for fabrics 1,3 and 4, but with most of the fibers at $45^{\circ}$ to the flow for fabrics 2 and 5 .

For all conditions the fabric canopies were suspended above the surface by using two tapered halfround dowels mounted on either side of the test area, as shown schematically in Figures 7, 8, and 9. The dowels effectively created acoustically silent 4-mm high ridges running down either side of the test area. The $25 \mathrm{~mm}$ long tapered sections of the dowel upstream and downstream of the test area produced short sections of fabric ramp at the leading and trailing edges of the test areas. Draping the fabrics over these dowels, tensioning the fabric spanwise and then taping the fabrics to the flow surface outside of the test area enabled a measure of control of the fabric height. An estimated tension of 5-10 $\mathrm{N}$ was distributed in the spanwise direction when the fabrics were mounted above the plate, and slight tension was distributed in the streamwise direction to eliminate any wrinkling of the fabric. It was observed that the shapes of the fabrics' pores were not significantly influenced by the application of this tension.

\subsection{Unidirectional Canopies}

Unidirectional canopies (essentially fabric canopies with fibers only aligned with the flow direction) were also tested. These were composed of fishing line (forming the fibers) suspended from a pair of 3D- 
printed, smoothly curved, $6.4 \mathrm{~mm}$ high humps (referred to as fiber supports) placed at the upstream and downstream edges of the same sandpaper roughness used in the mesh canopy experiment (Figure 10). The fiber supports were attached to an aluminum plate, and fishing line was wrapped around the entire plate and over the supports. To accommodate the increased thickness of this surface, a shaped metal transition, $305 \mathrm{~mm}$ in length, was used to gradually elevate the wall-jet $12.7 \mathrm{~mm}$ above the base plate (Figure 10). The transition, used previously by Awasthi et al. ${ }^{15}$, had the shape of a shallow inflection. The $0.7 \mathrm{~mm}$-high forward step at the leading edge of the transition was faired using $0.12 \mathrm{~mm}$ thick metal tape to eliminate it as a noise source and to minimize its flow disturbance. When assembled in the wind tunnel, the result was a surface flat and continuous with the surrounding wall, with the exception of the streamlined supports at the leading and trailing edges of the test-portion of the surface and the unidirectional canopy formed between them.

Three complete test surfaces were manufactured, each with a different fishing line diameter. Table 3 gives the relevant parameters of the three canopies. The top surfaces of the supports used with each test surface were manufactured with evenly-spaced grooves so as to precisely accommodate the fishing line fibers wound over them and to set the fiber spacing so as to produce an open area ratio of $70 \%$. Figure 11 shows close-up views of each of the three canopies suspended above sandpaper roughness.

The tension used to wrap the fishing line was intended to be kept constant at around 4-8 $\mathrm{N}$ using a fishing reel with a calibrated line drag setting. This was successfully accomplished for canopies 1 and 2 . However, this proved more difficult than expected for the thickest fishing line (canopy 3). In this case, the thread was wrapped by hand while attempting to keep the tension in the fibers constant.

\subsection{Microphone Instrumentation}

Measurements of the far-field sound spectrum and directivity radiated by the roughness and fabric were made using a series of six 1/2-inch diameter Bruel and Kjaer (B\&K) Type 4190 microphones positioned in an arc $607 \mathrm{~mm}$ from the center of the roughness fetch with receiver angles between 55 and 129 degrees from the downstream surface. The microphone configuration is illustrated in Figure 12. All far-field data presented in this paper was collected from the microphone located at 129 degrees, as no additional conclusions could be drawn from the other microphones' data. Surface pressure fluctuations at the center of the test area were measured for all surface and flow conditions, either on the smooth wall or on the roughness substrate surfaces. During the unidirectional canopy experiments, surface pressure fluctuations were also measured $51 \mathrm{~mm}$ directly upstream and downstream of the center. Pressure fluctuations were sensed using a Sennheiser KE4-211 pinhole electret microphone with an attached 1/2$\mathrm{mm}$ pinhole covering. Phase and amplitude characteristics of the Sennheiser, with pinhole, were obtained by calibrating it against a B\&K Type 4138 microphone using a white noise source in an anechoic chamber. 


\section{Experimental Results and Discussions}

\subsection{The uncovered smooth and rough surfaces}

Figure 13 compares surface pressure spectra measured on the smooth wall and on the substrates of each of the rough surfaces. For the smooth wall, the surface pressure spectra display a form that is fairly typical of a wall jet. At low frequencies (less than about $1 \mathrm{kHz}$ in the present flows) spectral levels are relatively high, presumably because of the pressure footprint of the energetic large scale structures that populate the outer mixing-layer portion of the flow. At mid-range frequencies, the spectra have a slope of close to -0.8 , analogous to the overlap region seen in external flow boundary layers, and then at high frequencies the spectra show a rapid roll off associated with viscous attenuation. As the speed and Reynolds number of the flow increases, the spectra shift up and to the right as a result of the greater absolute energy in the turbulence, the greater convective speed over the surface pressure microphone, and the decreasing scale at which viscous dissipation becomes important.

The rough surfaces have different effects on this background form. Spectra measured at the center of the $3 \mathrm{~mm}$ hemispherical roughness fetch (Figure 13a) show only very slightly altered levels compared to the smooth wall, the most marked difference being a reduction of several decibels in levels at high frequencies. Because the roughness elements were somewhat widely spaced, with the surface pressure microphone located some distance from the nearest element, the microphone did not capture the full effect of the roughness elements on the surface pressure fluctuations, the majority of which is seen in the wake of each element. This does not happen with the sandpaper roughness (Figure 13b) which is a very effective turbulence producer and increases wall pressure fluctuations across the spectrum, particularly at high frequencies and low speeds.

Radiated noise spectra from the surfaces measured by the forward-most microphone are compared in Figure 14. Roughness noise is generated by the scattering of these pressure fields (or at least their parent wavenumber frequency spectra) from the uneven surface geometry. The acoustic spectra for the smooth wall, of course, include no such scattering effects and instead merely indicate the background noise levels in the facility as a function of flow speed. Devenport et al. ${ }^{13}$ identify these as result of jet noise generated at the nozzle exit. At speeds of $40 \mathrm{~m} / \mathrm{s}$ and above, the hemispherical rough surface produces discernable roughness noise above the background starting at about $700 \mathrm{~Hz}$ and extending to the upper limit of the measured frequency range at $20 \mathrm{kHz}$. The signal to noise ratio, however, remains quite low, never exceeding about $4 \mathrm{~dB}$. The sandpaper surface has sharp corners that serve as much more efficient scatterers. Signal levels are therefore much higher. Noise from the sandpaper roughness at $60 \mathrm{~m} / \mathrm{s}$ becomes audible at about $2 \mathrm{kHz}$ but by $20 \mathrm{kHz}$ is some $18 \mathrm{~dB}$ above the background.

\subsection{Fabric Canopies}


In this section acoustic and surface pressure fluctuation results are presented and discussed for the various smooth and rough surface conditions with the canopy coverings. Fabric 5, described above, will be the focus of this section for two distinct reasons. First, this canopy most closely mimics the characteristics of the owl-down canopy since it has the thinnest fibers which run approximately $45^{\circ}$ relative to the flow direction, while the other canopies have thicker fibers, some of which run perpendicular to the flow direction. Second, it became clear from the data gathered during this experiment that fabric 5 was the most effective in reducing the far-field noise and surface pressure fluctuations while minimizing additional noise from the canopy itself. We also focus our presentation on acoustic measurements made with the forward-most far-field microphone, at a receiver angle of $129^{\circ}$.

Figures 15 and 16 show the effects of installing fabric 5 over top of these different surfaces in terms of pressure fluctuations at the center surfaces and the sound recorded overhead. Considering first the surface pressure fluctuations for the smooth surface, it is evident that the canopy causes a slight increase in pressure fluctuation intensities at low frequencies $(<2 \mathrm{kHz}$ for $60 \mathrm{~m} / \mathrm{s})$, but a substantial reduction in pressure fluctuations at higher frequencies, particularly in the viscous roll-off region. At $9 \mathrm{kHz}$ the canopy causes about a $15 \mathrm{~dB}$ reduction in the surface pressure fluctuations. It is almost as though the canopy increases the effective viscosity, reducing the frequency at which the roll off occurs.

While the suppressing effect of the canopy on high-frequency surface pressure fluctuations is large with the smooth wall, it becomes overwhelming with the rough surfaces. Within the hemispherical roughness fetch pressure fluctuations at $60 \mathrm{~m} / \mathrm{s}$ are slightly increased below $800 \mathrm{~Hz}$ and reduced at higher frequencies, with the attenuation reaching some $25 \mathrm{~dB}$ at $9 \mathrm{kHz}$. The fabric covering over sandpaper produces very little amplification of low frequency pressure disturbances, but retains the approximate 25 $\mathrm{dB}$ attenuation near $10 \mathrm{kHz}$ at $60 \mathrm{~m} / \mathrm{s}$, as seen for the hemispherical roughness. Interestingly, the maximum attenuation appears to increase as the flow speed and the Reynolds number are reduced, reaching $30 \mathrm{~dB}$ around $1500 \mathrm{~Hz}$ with the hemispherical roughness at $20 \mathrm{~m} / \mathrm{s}$. This suggests that the canopy is able to exert the greatest viscous influence on the flow at lower speeds due to the lower Reynolds number of the canopy fibers. At this lowest speed, the estimated Reynolds number based on the filament diameter $(0.06 \mathrm{~mm})$ and the local undisturbed mean flow velocity is about 20, compared to 7 for the owl down fibers. Figure 17 shows a comparison with results obtained with the other canopy materials of Figure 6 covering the hemispherical roughness fetch. The result is an overall increase in the attenuation of surface pressure fluctuations as the open area ratio and the pore size are reduced.

As a final point of discussion regarding the attenuation of surface pressure fluctuations, Figure 18 shows scaled surface pressure spectra obtained with canopy 5 shrouding each underlying surface. The frequency scaling is based on the maximum local flow speed in the boundary layer $\left(U_{m}\right)$ and the boundary layer thickness $(\delta)$, while the magnitude scaling is obtained by normalizing the power spectral density levels $\left(G_{p p}\right)$ on the square of the dynamic pressure $\left(1 / 4 \rho^{2} U_{m}^{4}\right)$. As seen in the figures, the attenuation of surface pressure fluctuations starts at a non-dimensional frequency near one, suggesting 
that the canopies are having the majority of their effect on turbulent structures which would be located in the boundary layer portion of the wall-jet flow, as opposed to the much larger turbulent structures present in the upper shear-layer portion of the flow. This might be expected as the height of canopy (approximately $4 \mathrm{~mm}$ ) is well-embedded in the boundary layer (with thickness of approximately 16-17 $\mathrm{mm})$.

With the surface pressure fluctuations attenuated one might expect an effect upon the noise radiated from the rough surfaces, depending on the amount of sound produced by the canopy itself. The self-noise of the canopy can be judged in Figure 16a, which compares noise levels with the canopy draped over the smooth wall to background levels in the facility. Audible canopy noise is seen at high frequencies, above $5 \mathrm{kHz}$ at $60 \mathrm{~m} / \mathrm{s}$, with the signal-to-noise ratio becoming larger as the frequency is increased. As seen in Figure 19, comparison with results obtained using the other fabrics shows an increase in canopy self-noise with filament diameter and the degree to which the filaments are perpendicular to the flow. This is, of course, consistent with the stealth of the owl, given the fineness of the fibers that form its down and their alignment with the flow direction in the down canopy.

The canopy has a mixed effect on the sound radiated from the rough surfaces (Figures 16b and 16c). At mid-range frequencies the canopy actually reduces the roughness noise. Greater reductions are observed when the canopy is placed over sandpaper as compared to the hemispherical roughness case, and the largest attenuation is approximately $5 \mathrm{~dB}$ near $5 \mathrm{kHz}$ for the $60 \mathrm{~m} / \mathrm{s}$ case. At high frequencies the canopy self-noise increases far-field sound levels over the rough surface alone. The increase in sound levels is smallest for the sandpaper rough surface.

\subsection{Unidirectional Canopies}

The results of the fabric canopy experiment showed that, in general, far-field noise increased as the fabrics featured cross-threads placed at higher angles relative to the flow direction. As such, a new hypothesis was developed which stated that if these cross-threads were to be removed, leaving a canopy with only fibers oriented in the flow direction, then the majority of the canopy self-noise could be eliminated, while the noise-cancelling aspects of the canopy could be retained. This led to the development of a unidirectional canopy, featuring faired thread supports upstream and downstream of the roughness fetch. There was concern that the presence of these supports would substantially alter the flow over the sandpaper or canopy, thereby affecting the acoustic behavior of these items. Therefore, before presenting data for the effects of the unidirectional canopies, results are given that reveal the comparatively minor effects of the thread supports on the radiated noise and surface pressure field. Figure 20 shows the isolated effect of the thread supports compared to the background levels of the tunnel without roughness. The supports raised the far-field levels by approximately $1-2 \mathrm{~dB}$ above the background across the frequency spectrum. It is hypothesized that the majority of the far-field effect is 
due to scattering of the nozzle noise from the curved surface of the support, and not to any additional aerodynamic source.

Figure 20 also shows the effect of the supports on the surface pressure spectrum at the three streamwise locations. The most upstream location (Figure 20b) shows an increase in magnitude across the frequency range for all speeds, with the greatest increase at $20 \mathrm{~m} / \mathrm{s}$. This increase could be caused by local separation at the down-slope of the support, which decreases in severity with increasing Reynolds number. Interestingly, the center location (Figure 20c) shows almost no effect from the addition of the supports. The downstream location (Figure 20d) experiences an increase in low and mid frequency levels, which could be due to slight upstream influence of the downstream support. Overall, although there are some noticeable effects from the supports, the differences are minor and do not hinder the canopy measurements.

Figure 21 shows the effect of placing sandpaper roughness between the mounts, again without a canopy present. The near- and far-field effects are nearly identical to the effect of sandpaper seen previously (as in Figures 13 and 14). The presence of the mounts does not significantly alter the noise generation of the sandpaper, so the effects of adding a canopy should not be influenced by the support structure.

Figure 22a shows the far-field effects of stringing the thinnest fibers over the sandpaper roughness. At higher flow speeds, far-field sound levels were reduced by as much as $6 \mathrm{~dB}$ at higher frequencies. At low flow speeds, the far-field results are distorted somewhat by the noise floor of the microphones, but even so, a noise reduction is observed. Fortunately, these reductions are achieved without the large increases in high-frequency noise observed with the mesh canopies.

Figure 22 also shows the acoustic results for unidirectional canopies with different thread diameters, but the same open-area ratio of $70 \%$. As the diameter of the thread (and the spacing between the fibers) increases, the canopy appears to become less effective at reducing the far-field noise. This may be explained by the differences in the attenuation of the surface pressure fluctuations produced by adding the canopy in each case, shown in Figure 23. Figure 23 shows the difference in the measured surface pressure spectra on a $\mathrm{dB}$ scale for each of the three canopies, sensed by the microphone at the center of the canopy. Several patterns emerge in looking at the complete data set. There appears to be a strong Reynolds number effect on the attenuation. In general, as the flow speed over the canopy increases, the attenuation decreases, especially for the thinnest canopy. For the case of the thinnest canopy and a jet speed of $20 \mathrm{~m} / \mathrm{s}$, the attenuation is highest.

At a fundamental level, this result points to physical scale as the dominant factor in the effectiveness of the canopy. The distance between the fibers likely plays an integral role by determining the scale of eddies which may pass through the canopy unhindered, which may be distorted or broken up upon passing through, and which may stay above the canopy altogether. At an extreme level, one could think of a canopy with many microscopic fibers, with microscopic areas between them. In this case, even with 
the same open area ratio of $70 \%$, the fibers would physically prevent all but the very smallest turbulent eddies to pass through unhindered, and viscous effects would dominate at this scale to dampen these eddies. This situation closely resembles that of owls' wings, and their slow flight speeds and microscopic hairs likely combine to produce extremely efficient pressure fluctuation attenuation at the surface.

Another pattern seems to be that the attenuation develops as it moves downstream. This trend is seen for all three canopies, but is most evident in the data corresponding to canopy 3 . Figure 24 shows the surface pressure attenuation beneath canopy 3 sensed by each of the three microphones underneath. This may be the sign of a pseudo-viscous damping mechanism which influences the flow as it moves downstream beneath the canopy. This damping mechanism may be the result of the turbulent eddies' interactions with the canopy itself, or the fact that the small-scale eddies beneath the canopy no longer receive energy from any large-scale turbulent eddies such that any remaining turbulent energy is quickly dissipated, or a combination of these two phenomena.

Figure 25 shows the comparison of far-field noise produced with and without sandpaper roughness beneath canopy 1 . Strangely, the effect of the sandpaper seems to be minimal. The levels are only about $1-2 \mathrm{~dB}$ higher when sandpaper is included in the test, which is nearly within the uncertainty of the measurements. This means that the sandpaper is making very little noise at all while covered with the canopy, and any noise above background levels is due primarily to the canopy itself. This is consistent with the fact that the surface pressure fluctuations have been attenuated by the canopy, which means that relatively little turbulent energy is available to be scattered into the far-field by the roughness. However, the canopy itself can act as a scattering mechanism given its exposure to the undisturbed flow above.

\section{The Mixing Layer Instability as a Model of Surface Pressure Reduction}

We now wish to propose a tentative model to explain features of the reduction in unsteady surface pressure levels which result from introduction of the unidirectional canopy. We note that in the surface pressure fluctuation attenuation plots of Figure 23, the shapes of the curves are remarkably similar to each

other. In each case there is a definite maximum reduction, whose amplitude decreases with $U_{\text {jet }}$. Our inspiration for modelling this effect comes from studies of air flow over forest canopies (see references $[16,17]$ for a review), where it is well known that the drag of the canopy retards the lower portion of the atmospheric boundary layer and creates thin shear layers above the canopy, which undergo a classical mixing-layer instability [18]. We hypothesize that the introduction of the fabric canopy has the same effect on the wall-jet boundary layer.

The next step in our argument is to note that shear instability leads to exponential growth in the streamwise direction, but exponential decay in the direction transverse to the shear layer. This effect can be seen most simply in the case of Helmholtz instability of a vortex sheet: for a shear of strength $U$ at frequency $\omega$, the Kelvin-Helmholz wave has exponential spatial growth rate $\omega / U$ and decays with transverse distance at exponential decay rate $\omega / U$. The vortex sheet is of course unstable at all 
frequencies and its spatial growth rate is unbounded. However, a genuine, finite-thickness, shear layer will typically only be spatially unstable over a finite frequency range, and will typically have a definite frequency at which a maximum growth rate (and therefore by analogy with Kelvin-Helmholtz a maximum transverse decay rate) occurs. These features are very much in tune with the features of the experimental results described in the first paragraph. Our hypothesis for the effect of the canopy is therefore as follows: the canopy introduces a mixing layer, which leads to instability waves (as nascent turbulent eddies), which, due to the presence of the canopy, are lifted higher above the solid boundary than they would have been without the canopy present. The pressure footprint of these eddies on the solid surface is therefore substantially reduced, by both the transverse exponential decay effect of the instability wave and by their greater stand-off from the wall.

In order to investigate our hypothesis further, we now consider the stability of a simple linear shear layer above the canopy, and compute the spatial growth rates of the system for varying frequency, which we will then use to infer the surface pressure response. Specifically, we investigate the spatial instability of the linear mixing layer, with a base flow. Velocities and lengths are non-dimensionalized in terms of the free-stream velocity $U_{s}$ and the layer thickness $\delta$. In terms of the wall-normal coordinate $y$, the mixing layer velocity profile is written as

$$
U(y)=\left\{\begin{array}{cl}
0, & y<0, \\
\frac{U_{s} y}{\delta}, & 0 \leq y \leq \delta, \\
U_{s}, & y>\delta .
\end{array}\right.
$$

To determine the spatial stability of the base flow $U(y)$ we first assume wavelike, two-dimensional velocity and pressure perturbations of the form

$$
[\tilde{u}(x, y, t), \tilde{v}(x, y, t), \tilde{p}(x, y, t)]=[u(y), v(y), p(y)] e^{i k x-i \omega t}
$$

Here $x$ is the streamwise coordinate, $\omega \in \mathbb{R}$ is the temporal frequency, $k \in \mathbb{C}$ is the streamwise wavenumber, $\tilde{u}$ and $\tilde{v}$ are the streamwise and wall-normal velocities, respectively, and $\tilde{p}$ is the pressure perturbation. Perturbations of this form satisfy the reduced Rayleigh equation

$$
\left(D^{2}-k^{2}\right) v=0
$$

where $D=d / d y$. This simplification allows us to determine an exact dispersion relation $d(\omega, k, b, \delta)$. Assuming a wall-normal velocity perturbation profile which satisfies the Rayleigh equation in each of the three mean profile regions gives 


$$
v(y)=\left\{\begin{array}{cl}
A e^{k y}, & y<0, \\
B e^{-k y}+C e^{k y}, & 0 \leq y<\delta, \\
D e^{-k y}, & y>\delta,
\end{array}\right.
$$

for $\operatorname{Re}\{k\}>0$. We impose continuity of the wall-normal velocity at $y=\delta$. At $y=0$ we introduce the following condition to account for the disruption of wall-normal momentum caused by the canopy

$$
v_{+} / v_{-}=\gamma, \quad 0 \leq \gamma \leq 1
$$

In equation (5.5), $v_{+}$and $v_{-}$denote the wall-normal velocities above and below the canopy at $y=0$, respectively. For $\gamma=0$ we have $v_{+}=0$, and the canopy behaves as a solid wall. For $\gamma=1$ we have $v_{+}=v_{-}$and there is perfect continuity across the canopy. Therefore $\gamma$ acts as a homotopy parameter between these two cases. We thus identify $\gamma$ as a parameterization of the open-area ratio of the canopy. Since this quantity is held constant at 0.7 for all canopies experimentally tested, we fix $\gamma=0.7$ in our calculations, unless otherwise specified.

In addition to the conditions on $v$ at $y=0, \delta$, we impose continuity of the pressure perturbation $p$ at the same wall-normal locations $y=0, \delta$. Pressure continuity requires, with $s=0, \delta$

$$
\left(\omega-k U_{s}\right) k v^{\prime}+\left.k U_{s}^{\prime} v\right|_{y=s+}=\left(\omega-k U_{s}\right) k v^{\prime}+\left.k U_{s}^{\prime} v\right|_{y=s-},
$$

The application of the above conditions allows us to eliminate the amplitudes $A, B, C, D$ and derive a nonlinear dispersion relation for complex $k$. The two conditions at $y=0$ give

$$
\begin{gathered}
B+C=\gamma A \\
B\left(U_{s}-\omega \delta\right)+C\left(U_{s}+\omega \delta\right)=\omega \delta A .
\end{gathered}
$$

The conditions at $y=\delta$ give

$$
\begin{gathered}
B e^{-k \delta}+C e^{k \delta}=D e^{-k \delta} \\
B e^{-k \delta}\left(U_{s}-\delta\left(\omega-k U_{s}\right)\right)+C e^{k \delta}\left(U_{s}+\delta\left(\omega-k U_{s}\right)\right)=\delta D e^{-k \delta}\left(\omega-k U_{s}\right) .
\end{gathered}
$$

On eliminating the amplitudes in equations (5.7) - (5.10), we arrive at the following dispersion relation, which we express in terms of the non-dimensional quantities $K=k \delta$ and $\Omega=\omega \delta / U_{s}$,

$$
d(\Omega, K, \gamma)=2(1+\gamma) \Omega^{2}-\left\{(1+\gamma)(1-2 K)-(1-\gamma) \mathrm{e}^{-2 K}-2 \gamma\right\} \Omega+\gamma\left(2 K-1+e^{-2 K}\right) .
$$


Complex solutions $K$ of $d(\Omega, K, \gamma)=0$ are found via Newton iteration. Unstable solutions of this equation exist in the range $0<\Omega<\gamma /(1+\gamma)$. We plot instability curves for various $\gamma$ in Figure 26.

The theoretical framework presented above is now used to reproduce, as far as possible, the experimentally observed SPL attenuation curves induced by unidirectional canopy 1 from Figure 23(a). In order to do this, we will make sensible choices for some of the parameters in our model which can be estimated, such as the jet speed. However, other parameters are harder to estimate, and instead we will try to tune the free parameters in order to try and fit the results. Therefore, we are not attempting, at this stage, to make a complete quantitative prediction without the need for any fitting. Rather, we wish to determine whether the instability model is capable of recovering the features seen in experiment.

En route to determining a comparison to the SPL data, we derive an expression for the pressure drop across the mixing layer. Using our expressions for the wall-normal eigenfunctions above, we arrive at the following relation

$$
\frac{p(\delta)}{p(0)}=\frac{2 \gamma(K-\Omega)^{2} e^{-K}}{\Omega\left(2(K-\Omega)-1+e^{-2 K}\right)}
$$

We then suppose that due to the wall-normal, exponential decay effect of the unstable wave, the SPL attenuation is related to the linear instability in the following manner

$$
\Delta \mathrm{SPL}=20 \log _{10}\left(e^{-\operatorname{Im}\{K\} D}\left|\frac{p(\delta)}{p(0)}\right|\right)
$$

The constant $D$ in equation (5.13) is the pressure instability length scale, i.e. the height above the canopy where the least stable linear wave is located in order to fix the level of attenuation observed in the experiment.

Experimental results indicate the velocity just above the canopy to be about one third of the total jet velocity. Identifying fluid velocity at the lower part of the mixing layer $(y=0)$ with the velocity just above the canopy, and assuming that in the turbulent dynamics shear layers exist which shear from the maximum jet velocity we choose the velocity drop across the mixing layer to be $U_{s}=U_{\text {jet }} / 3$. Frequencies $f$ in Hertz can then be mapped to non-dimensional frequencies using the relation

$$
\omega=\frac{2 \pi f \delta}{U_{\mathrm{s}}}
$$


We use the experimentally observed frequency range to determine the layer thickness $\delta$ for each jet velocity. Results of fitting SPL attenuation and the layer thicknesses are included in Figures 27 and 28.

The good agreement between the experimental and theoretical attenuation curves presented in Figure 27 suggests that the canopy instability model is capable, for suitably chosen parameter values, of capturing aspects of the pressure attenuation, specifically at higher jet velocities. The parameter values of the model are reported in Figure 28, where we see a trend of approximately constant layer thickness $\delta$, and decreasing length scale $D \delta$ with increasing jet velocity. From these results we can conclude that a finely structured mixing layer instability approximates the attenuation trend until a maximum reduction is reached at high frequency and any attenuation is shut off by some other mechanism. Elucidation of this latter mechanism is a matter for further research.

\section{Conclusions}

Many species of owl are able to hunt in effective silence, a feat which is believed to be linked to three unique physical attributes: a comb of evenly-spaced bristles along the wing leading-edge; a compliant and porous fringe of feathers at the trailing-edge; and a velvety down material distributed over the upper wing surface. This paper has focused on the last of these mechanisms which is being investigated through experiments made on surfaces that mimic some geometrical features of the owl-down, as well as theoretical analysis of the aeroacoustics of canopy coverings and of flexible hairs.

Microscope photographs of the down show that it consists of hairs that form a structure similar to that of a forest. The hairs initially rise almost perpendicular to the feather surface but then bend in the flow direction over to form a canopy with an open area ratio of about $70 \%$. Experiments have been performed to examine the aeroacoustic effects of vertical filaments and by the large open area ratio fabric canopy suspended above a surface. The canopy is found to dramatically reduce pressure fluctuations on the underlying surface, in a manner that is found to be consistent the theory of flows over and through vegetation. While the canopy can produce its own sound, particularly at high frequencies, the reduction in surface pressure fluctuations can reduce the noise scattered from an underlying rough surface, particularly in the mid-frequency range. The use of a canopy with fibers oriented only in the flow direction does not produce the high-frequency self-noise of the fabric canopies, but surface pressure fluctuations are still suppressed. The effectiveness of the canopies in reducing the surface pressure fluctuations and far-field noise is primarily dependent on the physical spacing between the fibers of the canopy, with more closely spaced fibers producing more signal attenuation.

\section{Acknowledgments}

The authors would like to thank the Office of Naval Research, in particular Drs. Ki-Han Kim and Woei-Min Lin, for their support under grants N00014-13-1-0244, N00014-14-1-0242, and N62909-12-17116 (NICOP). IAC and JWJ are grateful to Prof. Raymond Goldstein for access to his photography 
equipment to examine the structure of owl down barbules. The assistance of Mr. Nathaniel Ross, Mrs. Katelyn McCormick, Mr. Daniel Grohol, and Mr. Jeffrey Gomes in the preparation of the surfaces for wind tunnel testing and in the performance of those tests is much appreciated.

\section{References}

1. Graham, R. R., “The silent flight of owls,” J. R. Aero. Soc., Vol. 38, 1934, pp. 837-843.

2. Kroeger, R. A., Gruschka, H. D., and Helvey, T. C., "Low speed aerodynamics for ultra-quiet flight," Tech. Rep. AFFDL-TR-71-75, Air Force Flight Dynamics Laboratory, Wright-Patterson AFB, 1972.

3. Hersh, A. S., Soderman, P. T., and Hayden, R. E., "Investigation of Acoustic Effects of Leading-Edge Serrations on Airfoils," J. Aircraft, Vol. 11, No. 4, 1974, pp. 197-202.

4. Jaworski, J. W. and Peake, N., "Aerodynamic noise from a poroelastic edge with implications for the silent flight of owls," J. Fluid Mech., Vol. 723, 2013, pp. 456-479.

5. Jaworski, J. W. and Peake, N., "Parametric guidance for turbulent noise reduction from poroelastic trailing edges and owls," Proceedings of the 19th AIAA/CEAS Aeroacoustics Conference, Berlin, AIAA-2013-2007, 2013.

6. Lilley G. M., "A study of the silent flight of the owl," Proceedings of the 4th AIAA/CEAS Aeroacoustics Conference, Toulouse. AIAA Paper 1998-2340, 1998.

7. Bachmann, T., Klan, S., Baumgartner, W., Klaas, M., Schroder, W., and Wagner, H., "Morphometric characterization of wing feathers of the barn owl Tyto alba pratincola and the pigeon Columba livia", Frontiers in Zoology, Vol 4, No. 23, 2007.

8. Bachmann, T., Anatomical, morphometrical and biomechanical studies of barn owls' and pigeons' wings," Ph.D. dissertation, RWTH Aachen University, 2010.

9. Chen, K., Liu, Q., Liao, G., Yang, Y., Ren, L., Yang, H., and Chen, X., "The Sound Suppression Characteristics of Wing Feather of Owl (Bubo bubo)," Journal of Bionic Engineering, Vol. 9, 2012, pp. 192-199.

10. Klan, S., Bachmann, T., Klaas, M., Wagner, H., and Schroder, W., "Experimental analysis of the flow field over a novel owl based airfoil," Experiments in Fluids, Vol. 46, 2009, pp. 975-989.

11. Klan, S., Burgmann, S., Bachmann, T., Klaas, M., Wagner, H., and Schroder, W., "Surface structure and dimensional effects on the aerodynamics of an owl-based wing model," European Journal of Mechanics B/Fluids, Vol. 33, 2012, 58-73.

12. Alexander, W. N., "Sound from Rough Wall Boundary Layers", Ph.D. dissertation, Virginia Polytechnic Institute and State University, 2011. avail: http://scholar.lib.vt.edu/theses/available/etd10112011-085924/

13. Devenport, W. J., Grissom, D. L., Alexander, W. N., Smith, B. S., and Glegg, S. A. L., 2011, "Measurements of roughness noise", J Sound Vib., Vol. 330,No. 17, pp. 4250-4273.

14. Wygnanski, I., Katz, Y., and Horev, E., "On the applicability of various scaling laws to the turbulent wall jet”, J. Fluid Mech., Vol. 234, 1992, pp. 669-690. doi: 10.1017/S002211209200096X

15. Awasthi, M., Bryan, B., Devenport, W. and Glegg, S., 2013, "Sound Radiation from Rounded Steps and Gaps", Proceedings of the 19th AIAA/CEAS Aeroacoustics Conference, Berlin. AIAA-20132048.

16. De Langre, E., 2008. "Effects of wind on plants," Annual Review of Fluid Mechanics, 40: 141-168

17. Finnigan, J., 2000. "Turbulence in plant canopies," Annual Review of Fluid Mechanics, 32:519-571

18. Raupach M. R., Finnigan J. J., Brunet Y. 1996. "Coherent eddies and turbulence in vegetation canopies: the mixing-layer analogy," Bound. Layer Meteorol. 78:351-382 
List of figure titles

Figure 1. Feathers examined: (a,b) Eurasian eagle owl (Bubo bubo) (c) great gray owl (Strix nebulosi) (d) snowy owl (Bubo scandiacus)

Figure 2. Cross section views of feathers from (a) a great gray owl and (b) a snowy owl.

Figure 3. Planform views at different depths. (a) Impermeable base layer, (b) middle layer, (c) top layer or 'canopy' (great gray owl).

Figure 4. Side-view schematic of the Virginia Tech Anechoic Wall Jet Wind Tunnel. All dimensions shown are in $\mathrm{mm}$.

Figure 5. (a) Hemispherical and (b) 20-grit sandpaper rough surfaces.

Figure 6. Images of the fabrics used. Fabrics were mounted so that the flow direction was top to bottom as shown in these figures. Note the diagonal orientation of the threads in the fabric 5 , the finest mesh.

Figure 7. Schematic view of fabric canopy suspended above smooth plate, with surface pressure microphone at center. The grey area outside the tapered dowels was covered with tape to restore a smooth surface.

Figure 8. Schematic view of fabric canopy suspended above hemispherical roughness.

Figure 9. Schematic view of fabric canopy suspended above sandpaper roughness.

Figure 10. Schematic view of unidirectional canopy suspended above sandpaper roughness. (a) Top View (b) Side View

Figure 11. Unidirectional canopies suspended above sandpaper roughness: (a) canopy 1, (b) canopy 2, and (c) canopy 3.

Figure 12. Schematic, side view of microphone instrumentation in wall-jet tunnel.

Figure 13. Surface-pressure spectra measured with the clean wall (solid symbols) and with the (a) hemispherical and (b) sandpaper rough surfaces (open symbols). Spectral levels are $1 \mathrm{~Hz}$ bandwidth SPL and are shown for different jet exit velocities $U_{j}$

Figure 14. Background far-field noise measurements made with the clean wall (solid symbols) and with the addition of roughness noise from the (a) hemispherical and (b) sandpaper rough surfaces (open symbols). Spectral levels are $1 \mathrm{~Hz}$ bandwidth SPL and are shown for different jet exit velocities $U_{j}$

Figure 15. Surface pressure fluctuations measured with fabric canopy 5 shrouding (a) the smooth wall, and the (b) hemispherical and (c) sandpaper rough surfaces (open symbols). Solid symbols show pressure fluctuation spectra recorded for each of these surfaces, respectively, without canopy.

Figure 16. Noise measurements made with fabric canopy 5 shrouding (a) the smooth wall, and the (b) hemispherical and (c) sandpaper rough surfaces (open symbols). Solid symbols show sound levels recorded for each of these surfaces, respectively, without canopy.

Figure 17. Attenuation of surface pressure fluctuations for each canopy over $3 \mathrm{~mm}$ roughness at $U_{j}=60$ $\mathrm{m} / \mathrm{s}$.

Figure 18. Scaled surface pressure fluctuations measured with fabric canopy 5 shrouding (a) the smooth wall, and the (b) hemispherical and (c) sandpaper rough surfaces (open symbols). Solid symbols show pressure fluctuation spectra recorded for each of these surfaces, respectively, without canopy.

Figure 19. Comparison of far-field noise for each fabric suspended above a clean wall at $U_{j}=60 \mathrm{~m} / \mathrm{s}$, illustrating the self-noise of each fabric.

Figure 20. Measurements of (a) far-field sound and surface pressure measurements from (b) upstream, (c) middle, and (d) downstream microphones showing the effects of adding the mounts (open symbols) to the clean configuration (solid symbols).

Figure 21. Measurements of (a) far-field sound and surface pressure measurements from (b) upstream, (c) middle, and (d) downstream microphones showing the effects of adding sandpaper roughness (open symbols) to the canopy mounts (solid symbols). 
Figure 22. Measurements of far-field noise from sandpaper covered with canopies (a) 1, (b) 2, and (c) 3 (open symbols) compared with those from uncovered sandpaper (solid symbols).

Figure 23. Surface pressure attenuation due to canopy (a) 1, (b) 2, and (c) 3 shrouding sandpaper roughness sensed by the center microphone.

Figure 24. Surface pressure attenuation due to canopy 3 shrouding sandpaper roughness sensed by microphone (a) 1, (b) 2, and (c) 3.

Figure 25. Measurements of far-field noise showing the difference in levels between the canopycovered sandpaper (solid symbols) and canopy without sandpaper (open symbols).

Figure 26. Mixing layer instability growth rates, for $\gamma=0.2,0.4,0.6,0.8,1$. The dashed curves are $\Omega=\gamma / 1+\gamma$

Figure 27. Comparison of experimentally measured surface pressure attenuation and theoretical prediction.

Figure 28. Plots of dimensional model parameters $\delta$ and $D \delta$ for given jet speeds. There is an approximate trend of constant layer thickness $\delta$, and decreasing length scale $D \delta$ with increasing jet velocity. 


\begin{tabular}{ccc}
$\begin{array}{c}\text { Jet Speed, } U_{j} \\
{[\mathrm{~m} / \mathrm{s}]}\end{array}$ & $\begin{array}{c}\text { Max Speed, } U_{m} \\
{[\mathrm{~m} / \mathrm{s}]}\end{array}$ & $\begin{array}{c}\text { BL Thickness, } \delta \\
{[\mathrm{mm}]}\end{array}$ \\
\hline 20 & 6.5 & 18.5 \\
\hline 30 & 10.1 & 17.2 \\
\hline 40 & 13.6 & 16.3 \\
\hline 60 & 17.3 & 15.7 \\
\hline 60 & 21.0 & 15.2 \\
\hline
\end{tabular}

Table 1 Boundary layer properties at $\boldsymbol{x}=1410 \mathrm{~mm}$

\begin{tabular}{cccccc} 
Fabric & Material & Open Area & $\begin{array}{c}\text { Pore Diameter } \\
{[\mathrm{mm}]}\end{array}$ & $\begin{array}{c}\text { Thread Diameter } \\
{[\mathrm{mm}]}\end{array}$ & $\begin{array}{c}\text { Tension Modulus } \\
{[\mathrm{N} / \mathrm{m}]}\end{array}$ \\
\hline 1 & Nylon & $75 \%$ & 3.9 & 0.3 & 508 \\
\hline 2 & Nylon & $64 \%$ & 3.1 & 0.4 & 555 \\
\hline 3 & Nylon & $76 \%$ & 2.1 & 0.2 & 1320 \\
\hline 4 & Polyester & $38 \%$ & 2.3 & 0.7 & 7930 \\
\hline 5 & Nylon & $70 \%$ & 1.6 & 0.06 & 117 \\
\hline
\end{tabular}

Table $\mathbf{2}$ Characteristics of fabric canopies

\begin{tabular}{ccc} 
Canopy & $\begin{array}{c}\text { Fiber Diameter } \\
{[\mathrm{mm}]}\end{array}$ & $\begin{array}{c}\text { Fiber Spacing } \\
{[\mathrm{mm}]}\end{array}$ \\
\hline 1 & 0.28 & 1.02 \\
\hline 2 & 0.56 & 1.82 \\
\hline 3 & 0.91 & 3.05 \\
\hline
\end{tabular}

Table 3 Characteristics of unidirectional canopies 

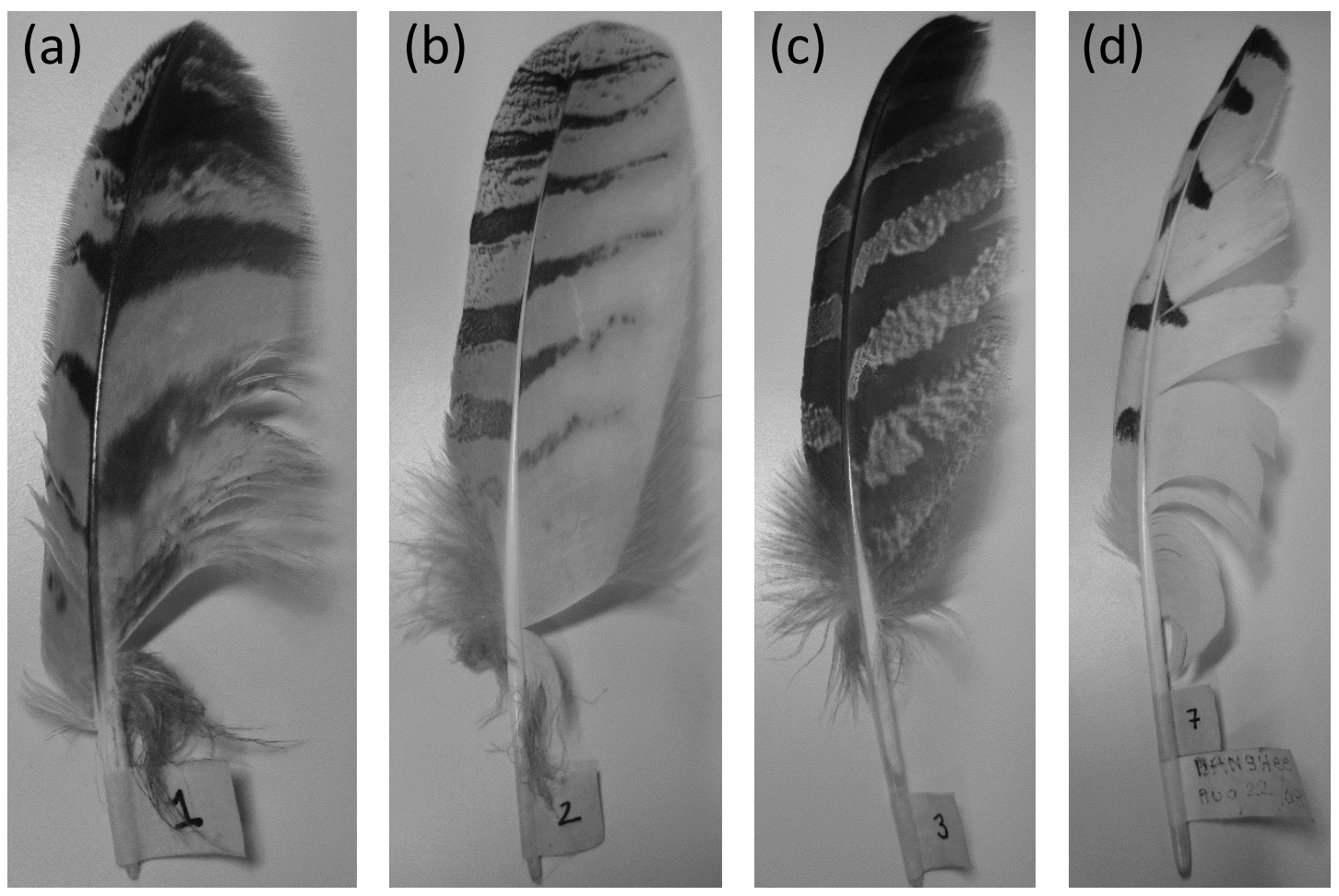

Figure 1. Feathers examined: (a,b) Eurasian eagle owl (Bubo bubo) (c) great gray owl (Strix nebulosi) (d) snowy owl (Bubo scandiacus) 

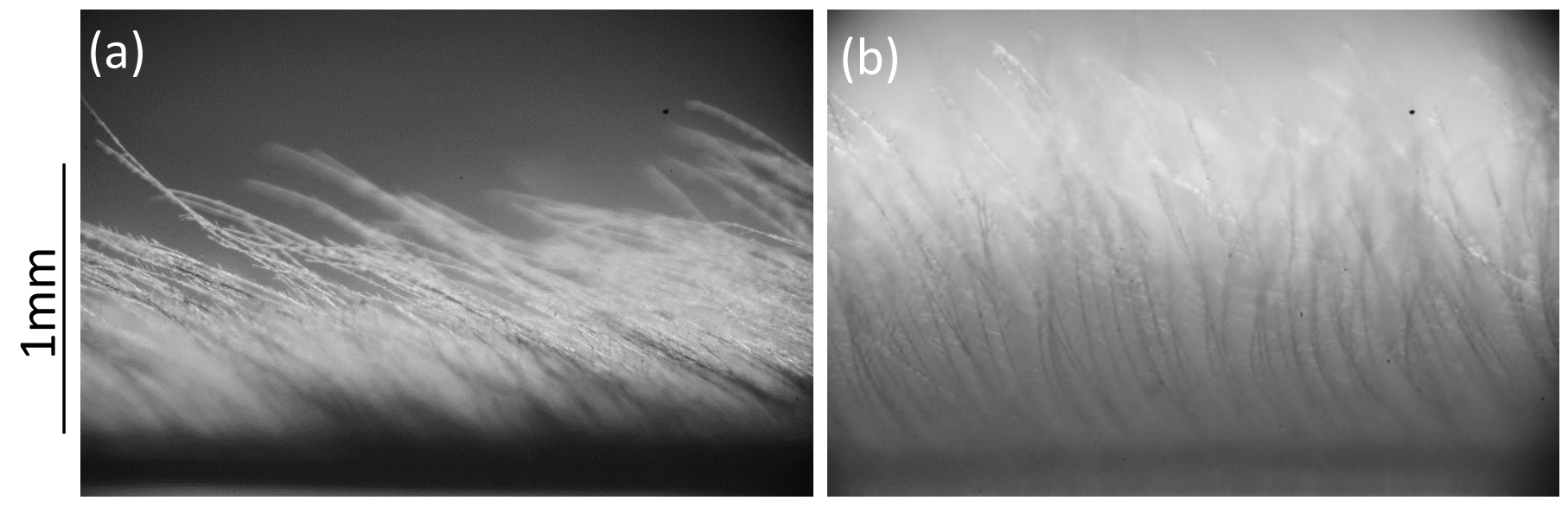

Figure 2. Cross section views of feathers from (a) a great gray owl and (b) a snowy owl.

Figure 2 

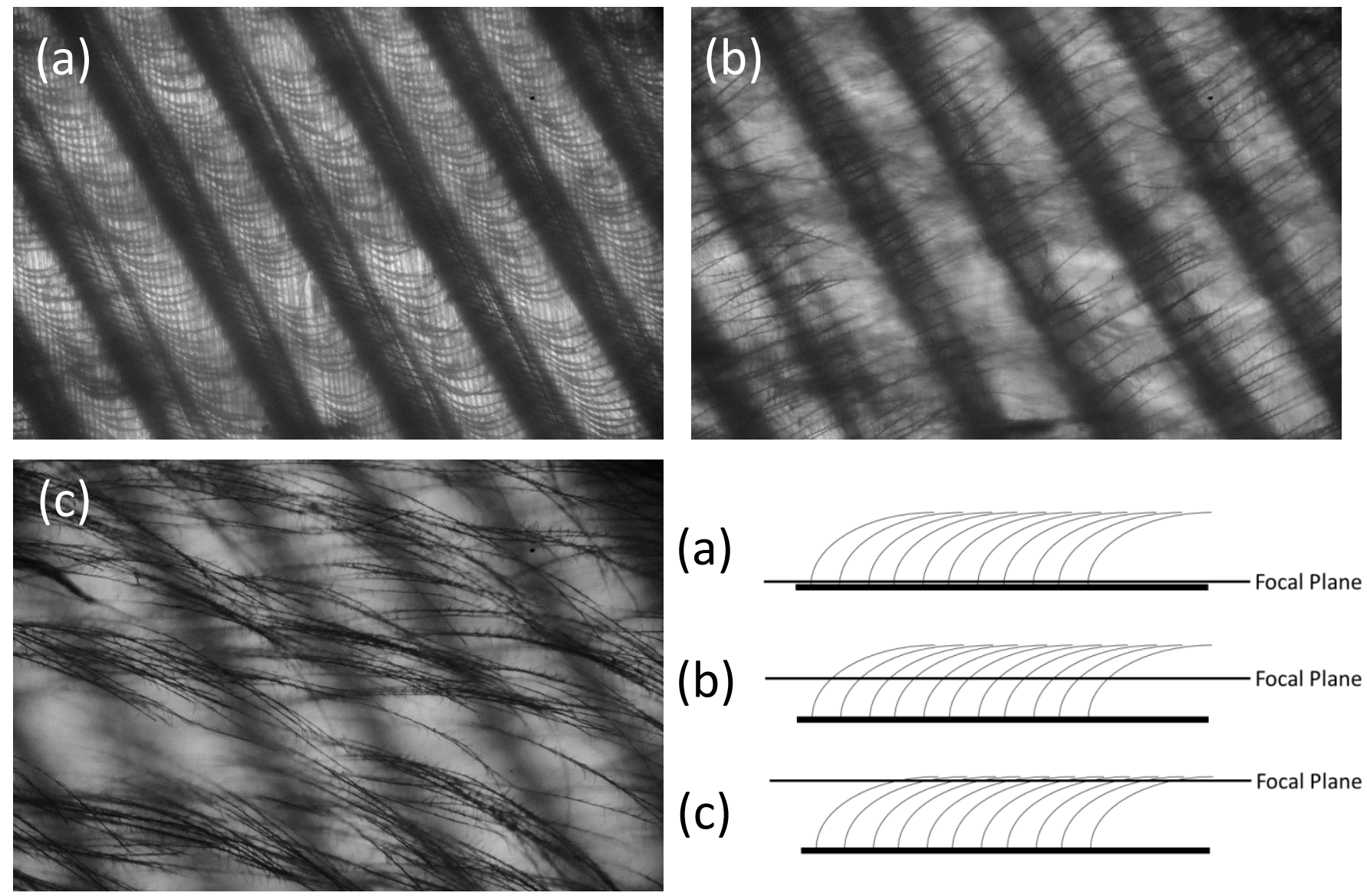

Figure 3. Planform views at different depths. (a) Impermeable base layer, (b) middle layer, (c) top layer or 'canopy' (great gray owl). 


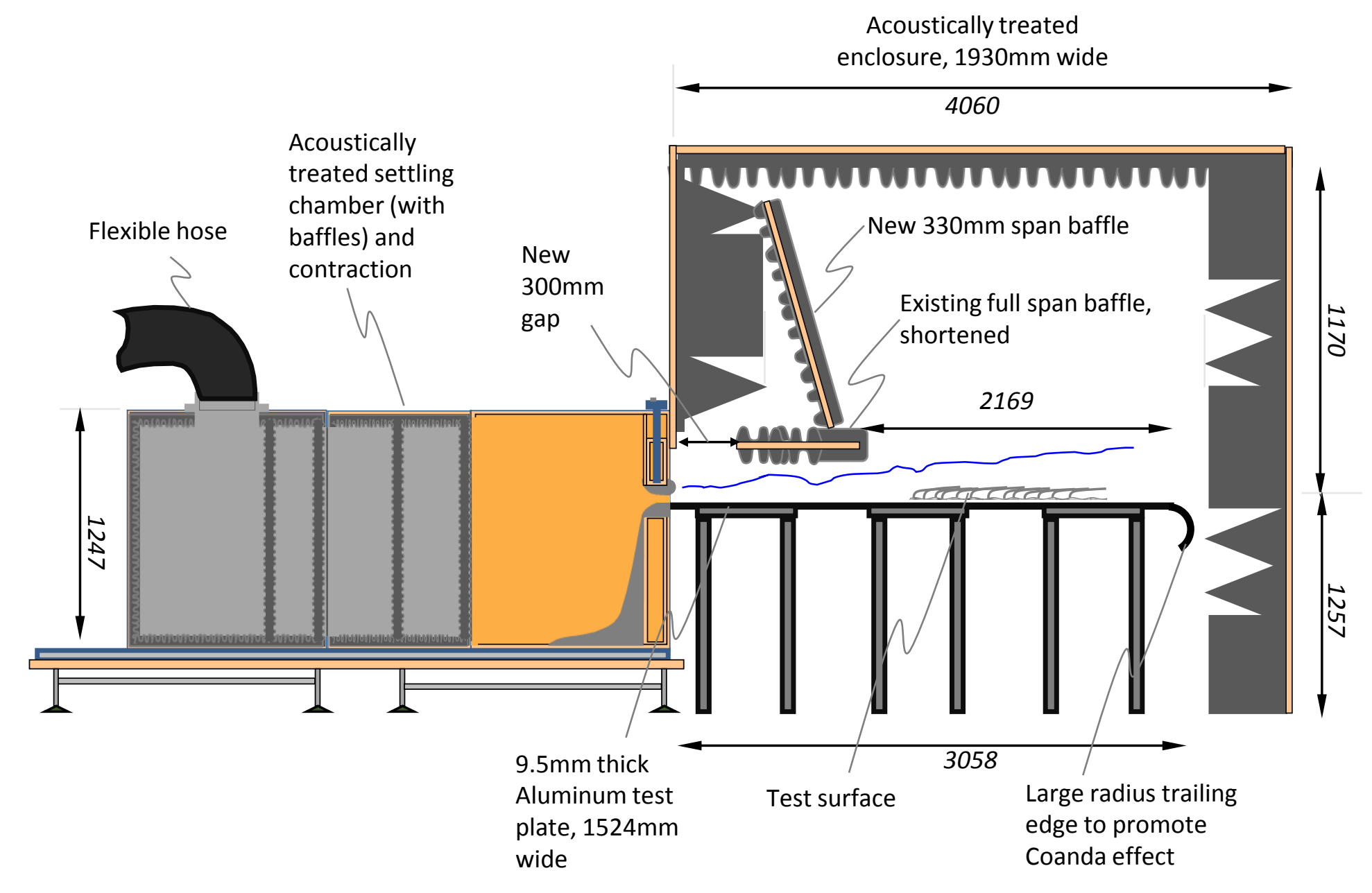

Acoustically
treated settling
chamber (with
baffles) and
contraction

Acoustically
treated settling
chamber (with
baffles) and
contraction

\section{.}




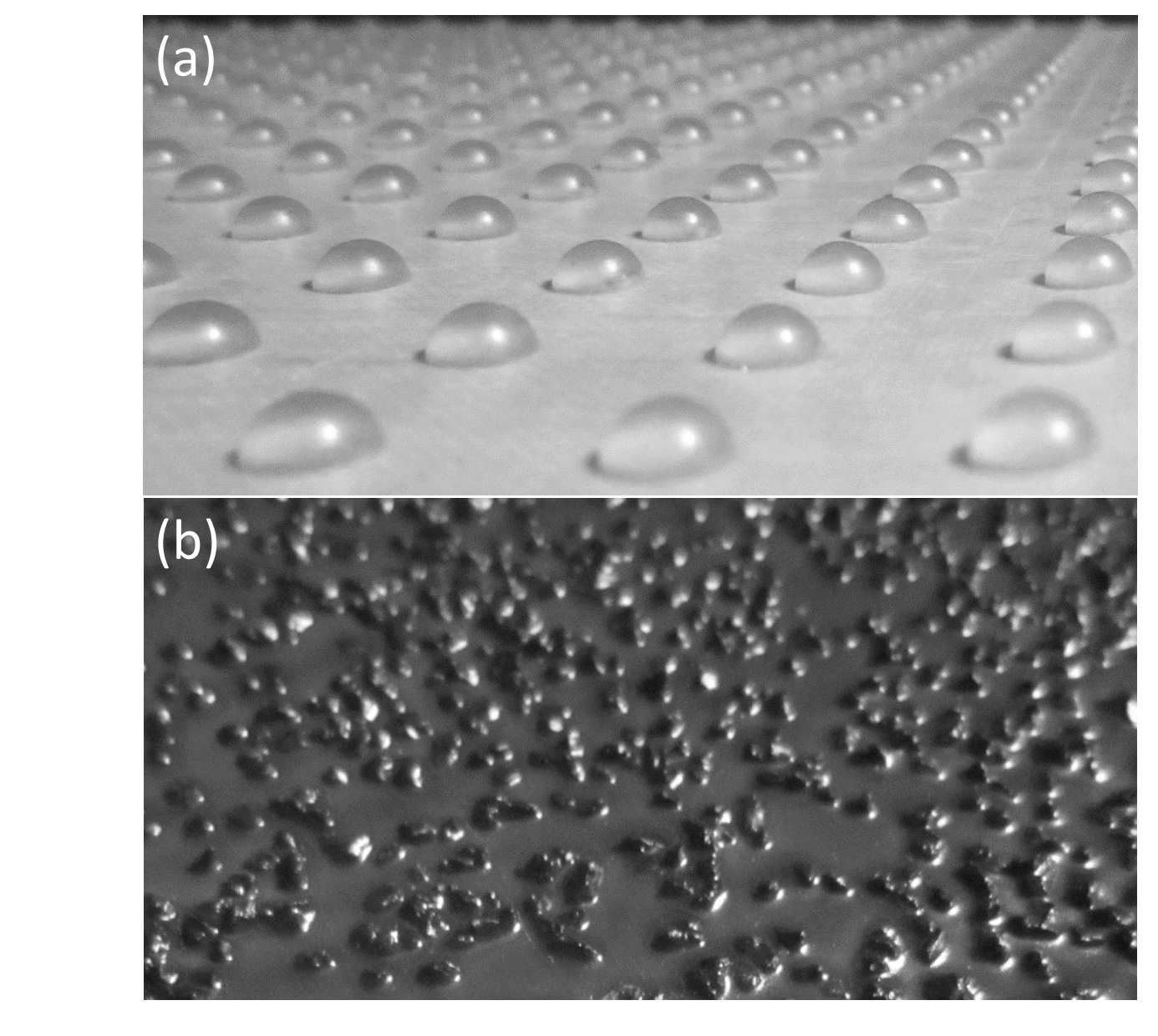

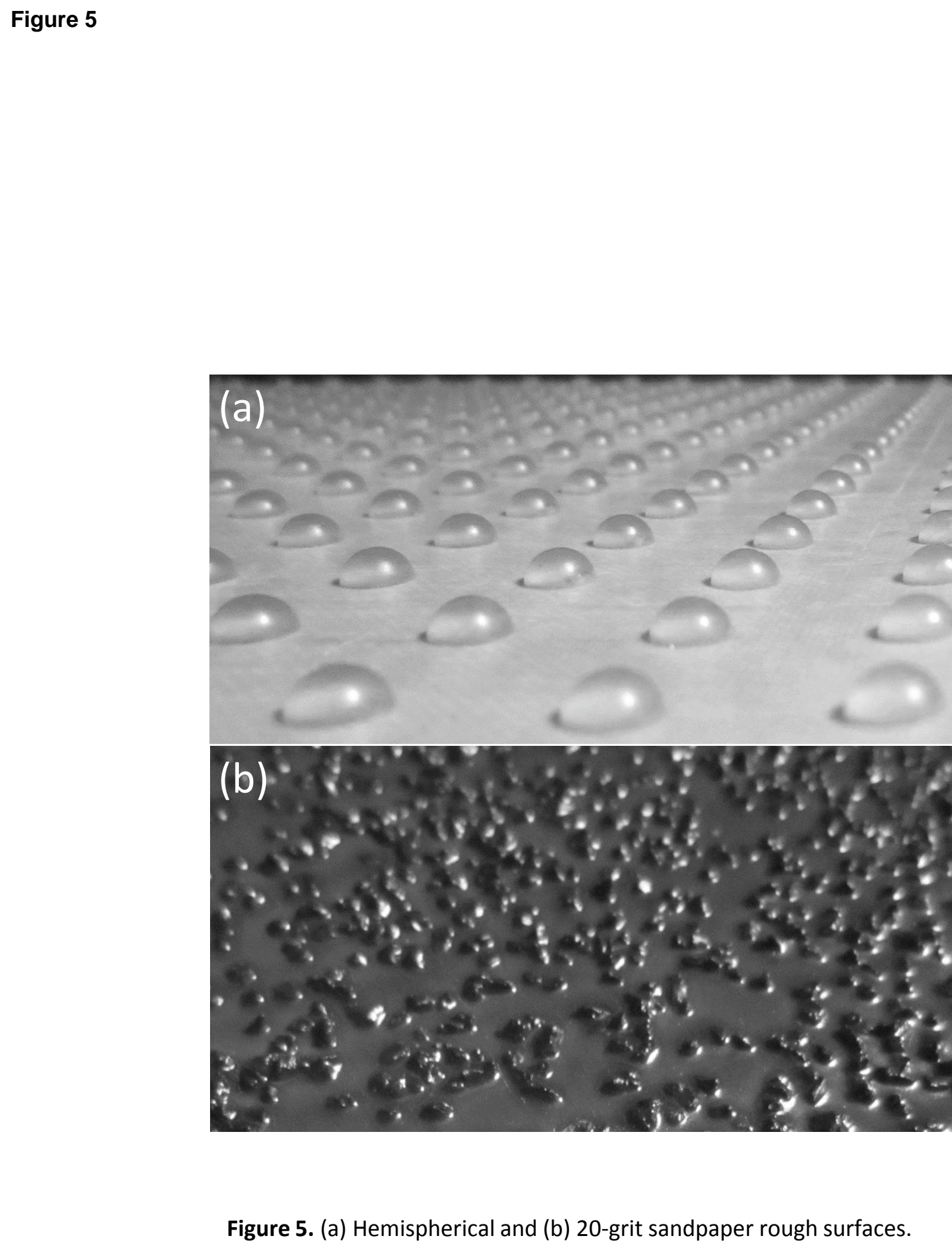

\author{
Figure 5. (a) Hemisphericaland (b) 20-grit sandpaper rough surfaces.
}

(2)

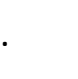

\title{
Figure
}

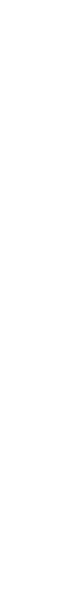



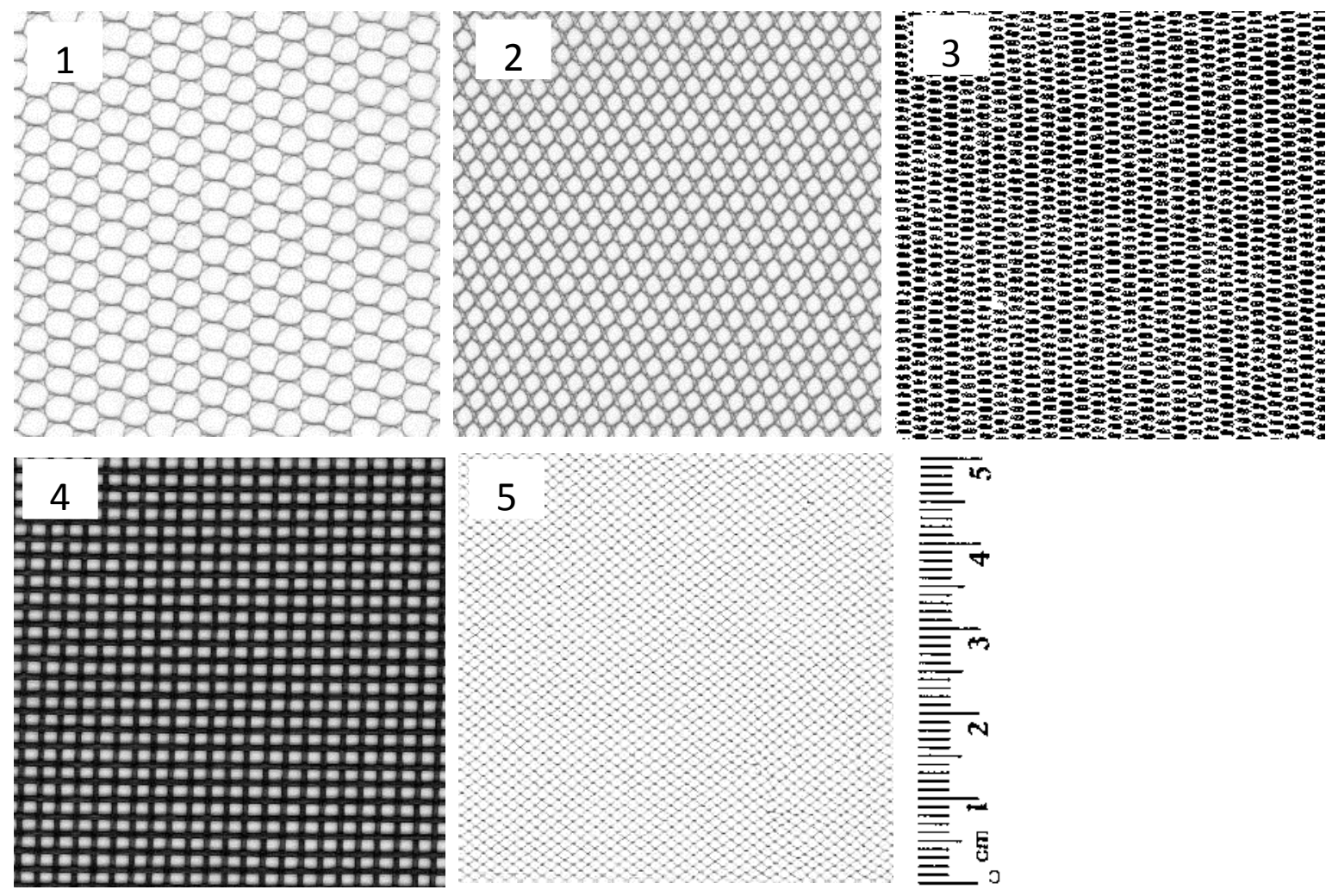

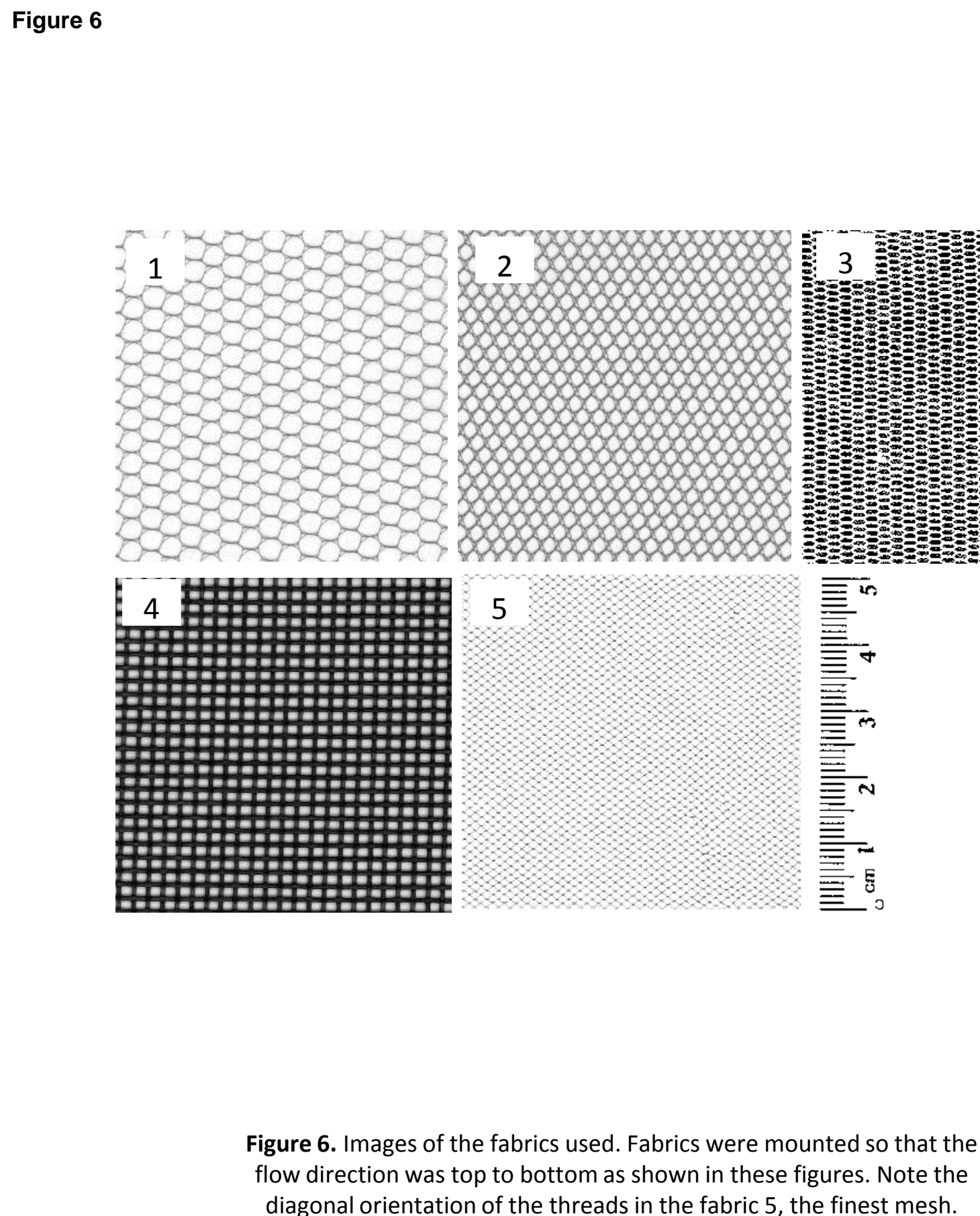

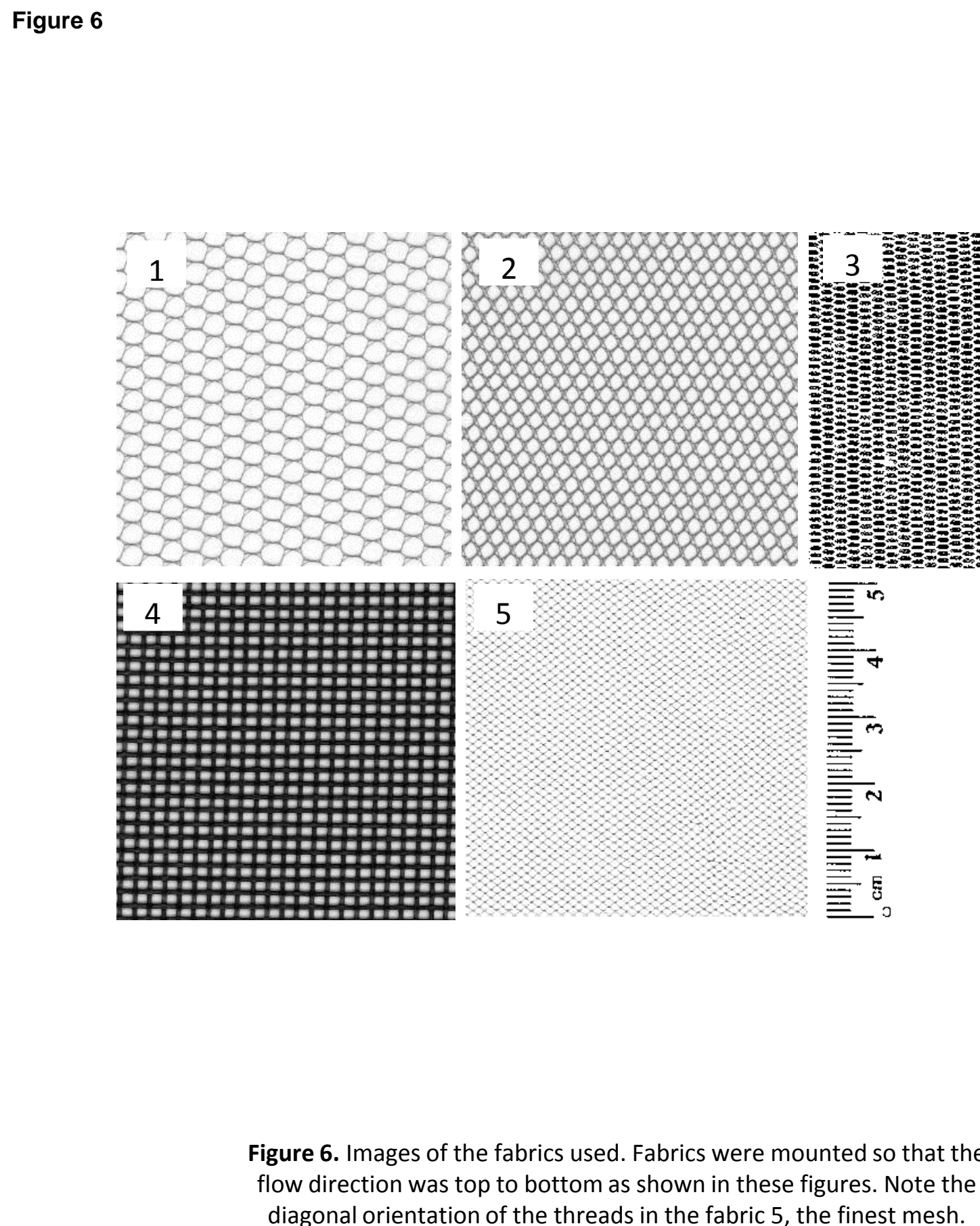

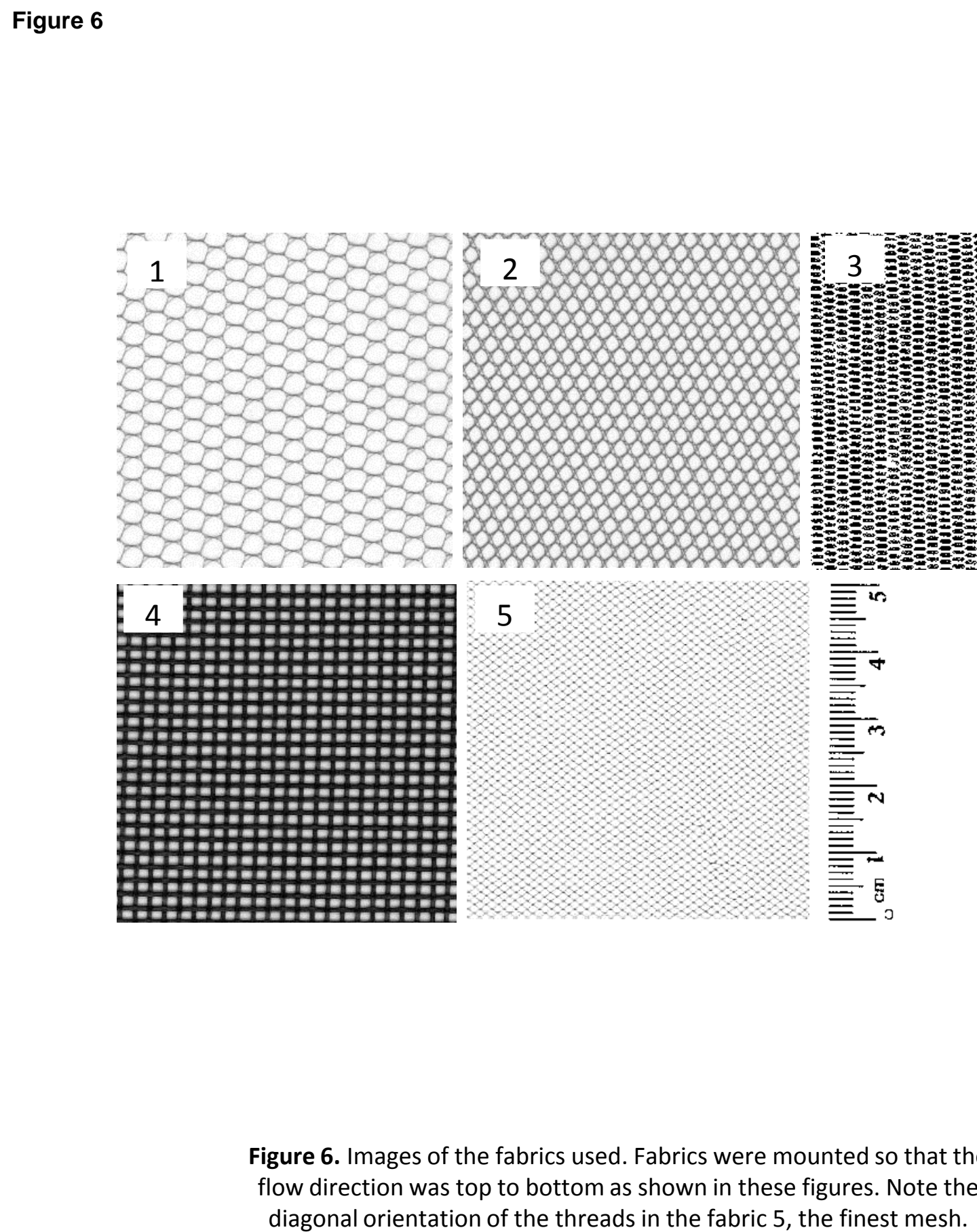

\author{
.
}

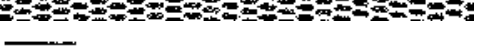




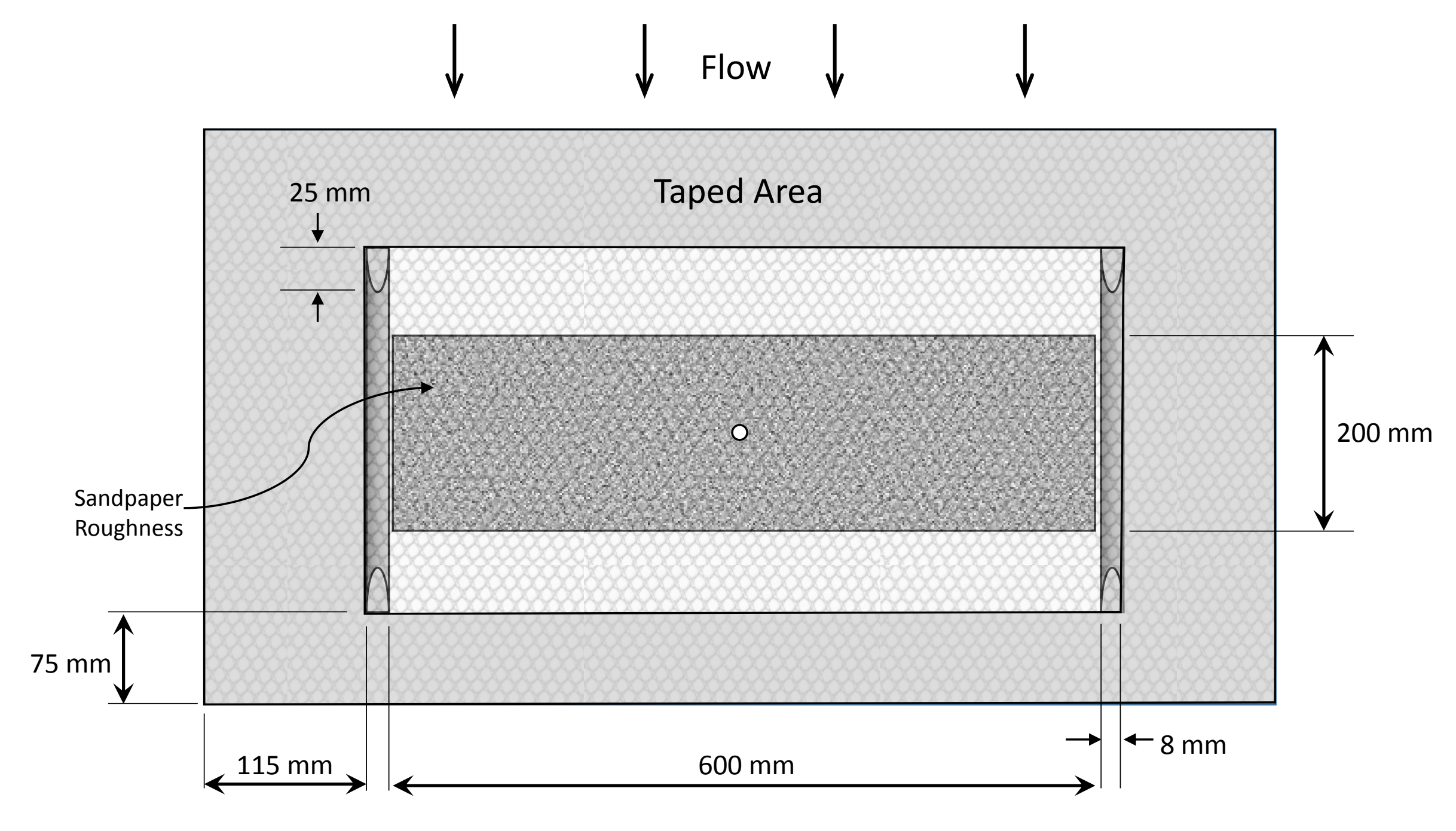

Figure 9. Schematic view of fabric canopy suspended above sandpaper roughness.

(a) 
(a)

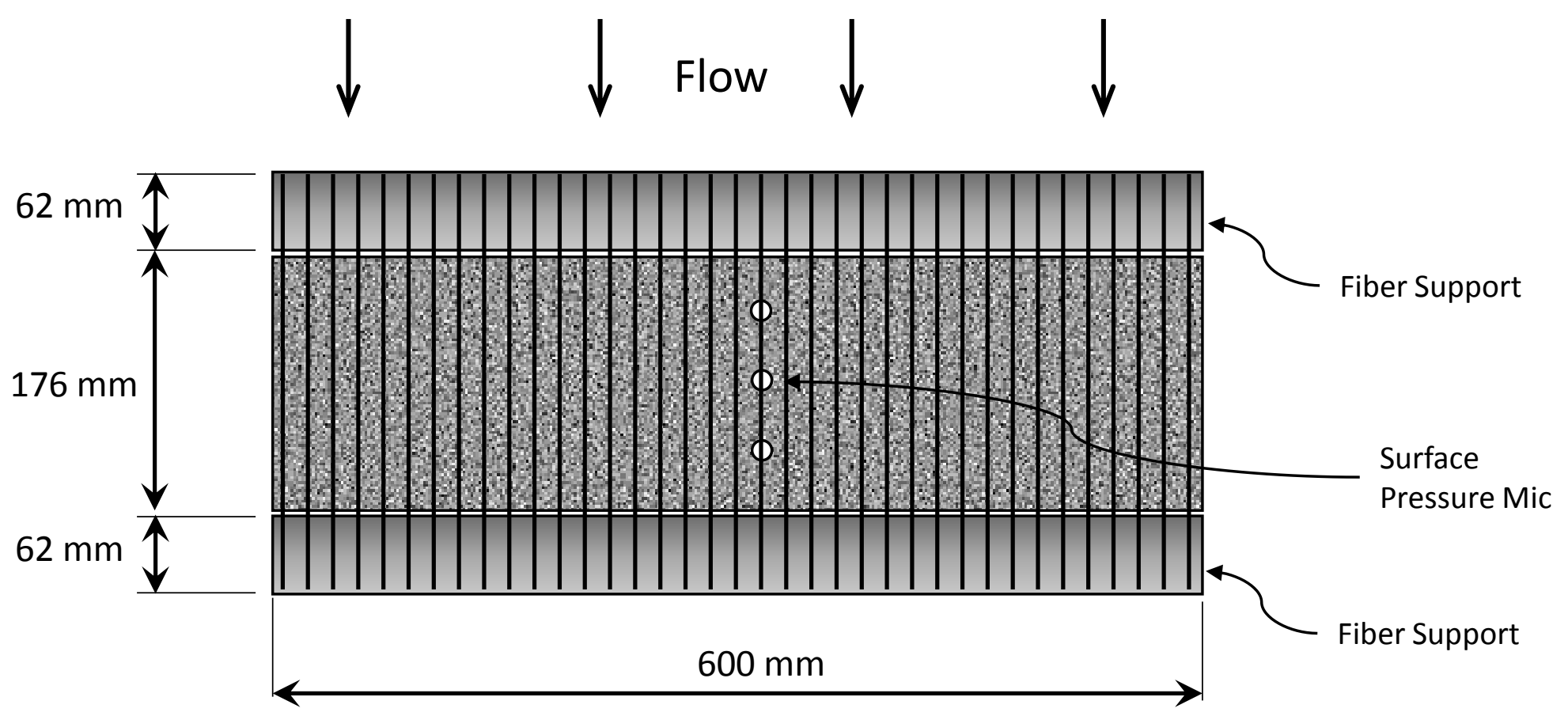

(b)

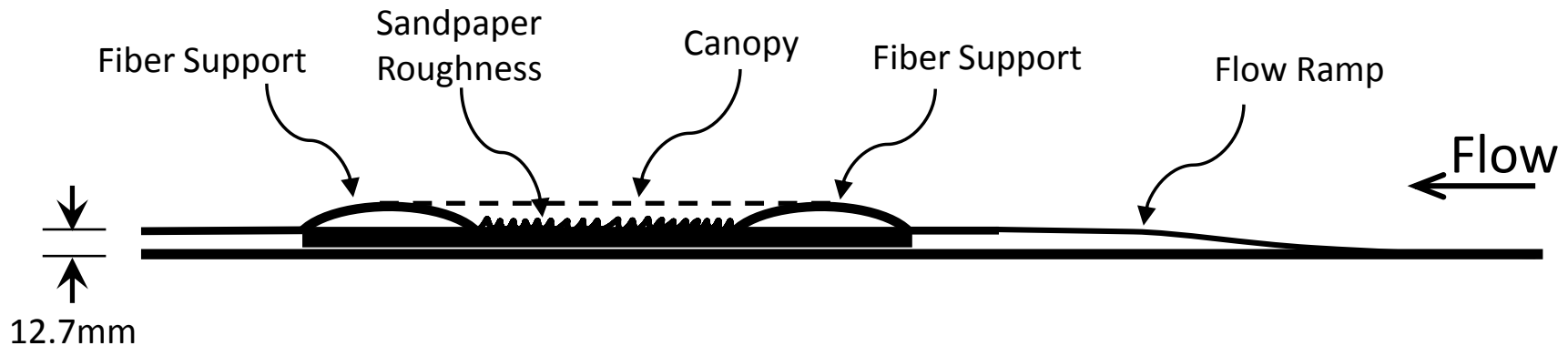

Figure 10. Schematic view of unidirectional canopy suspended above sandpaper roughness.

(a) Top View (b) Side View 

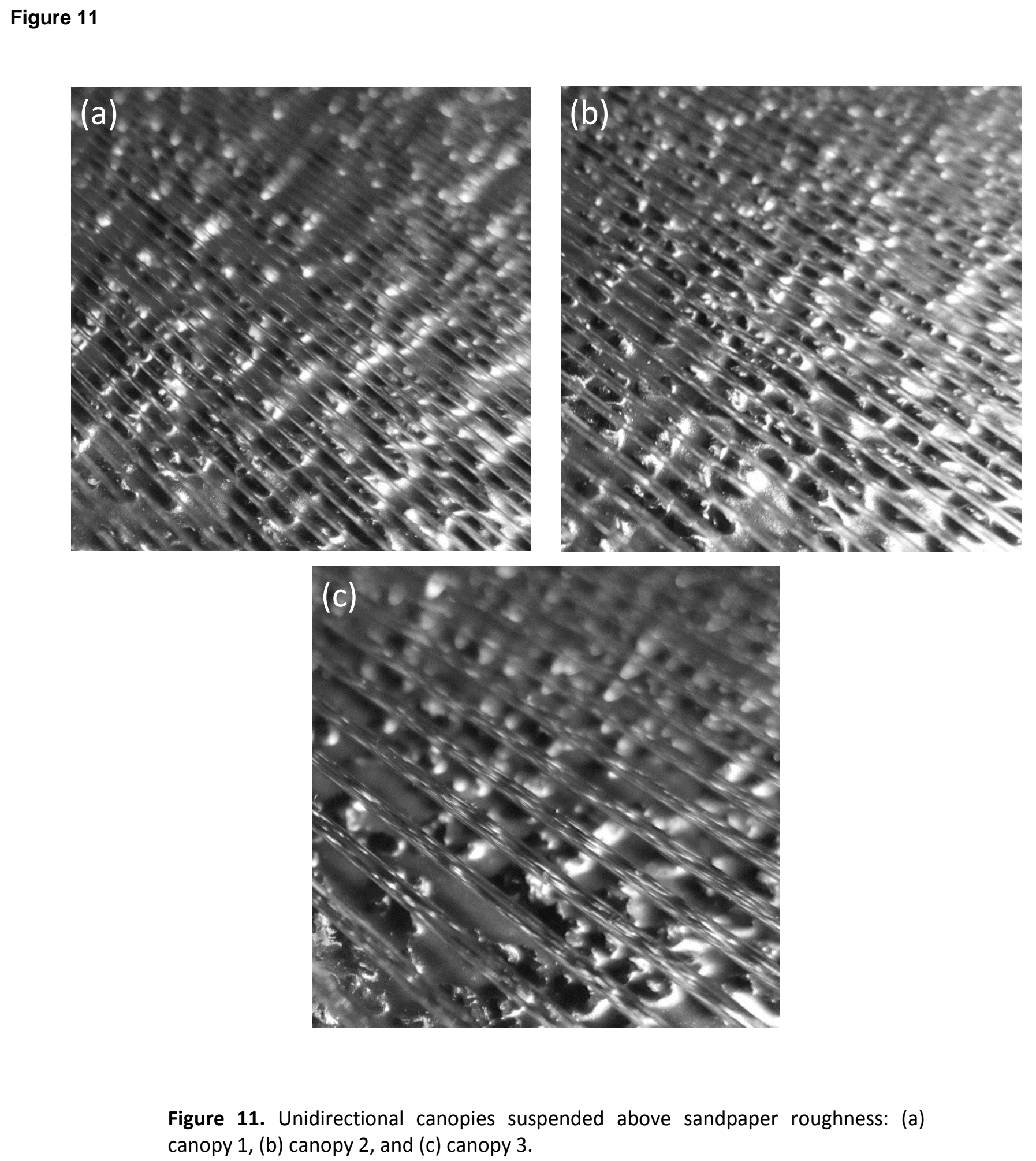

Figure 11. Unidirectional canopies suspended above sandpaper roughness: (a) canopy 1 , (b) canopy 2 , and (c) canopy 3.

\author{
canopy 1, (b) canopy 2, and (c) canopy 3.
}
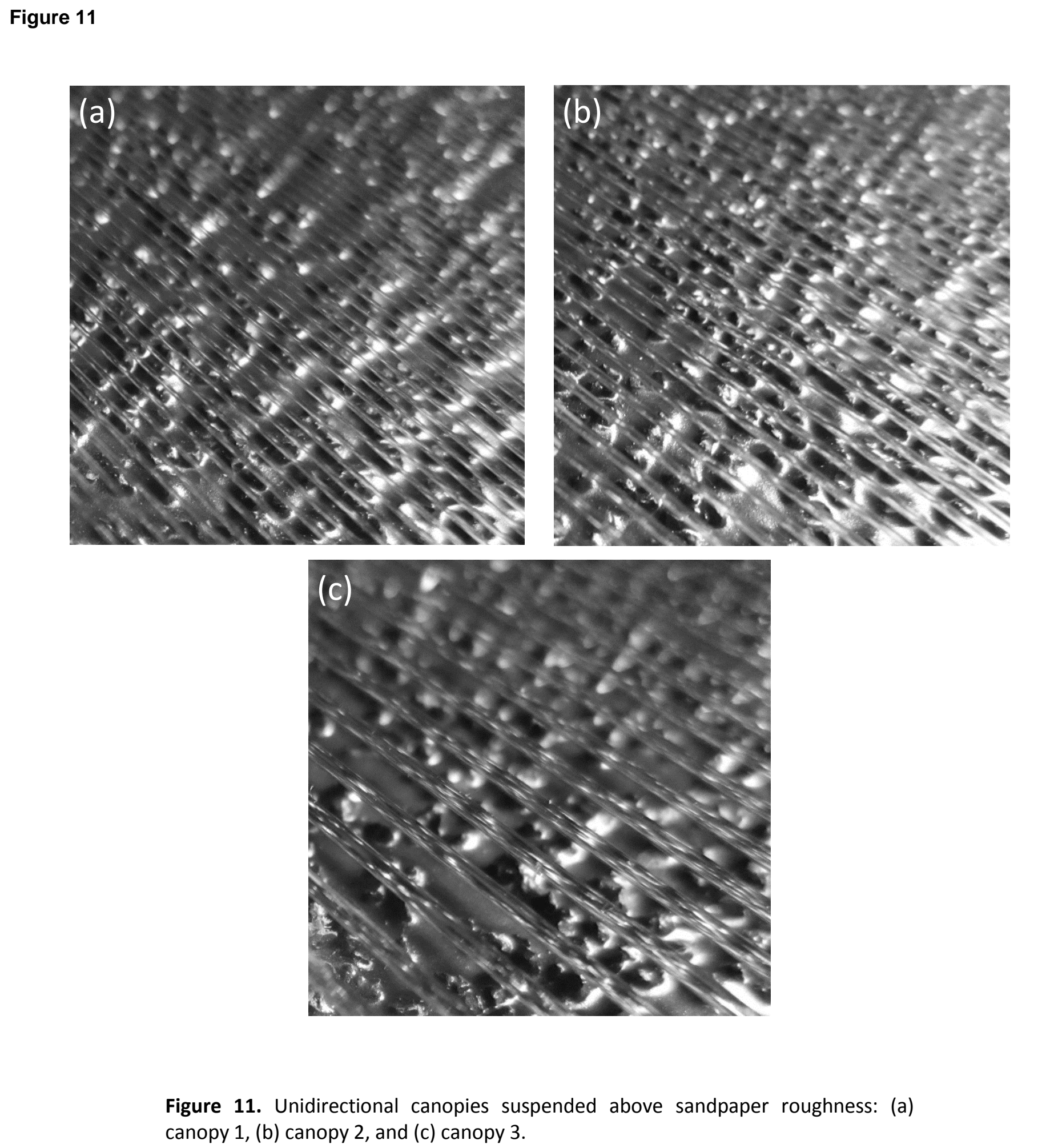

oughness: (a)

\author{
)
}




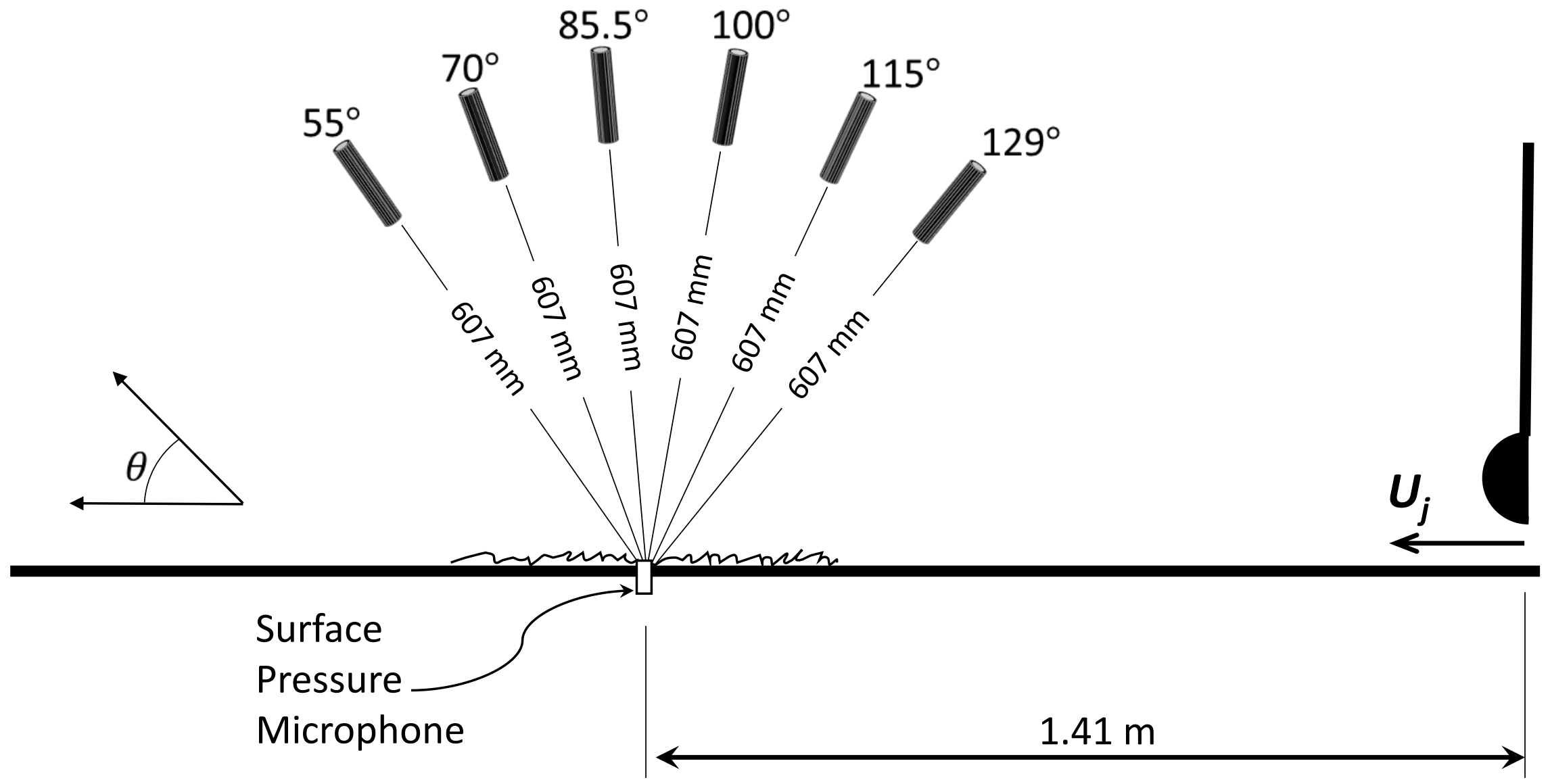

Figure 12. Schematic, side view of microphone instrumentation

in wall-jet tunnel. 

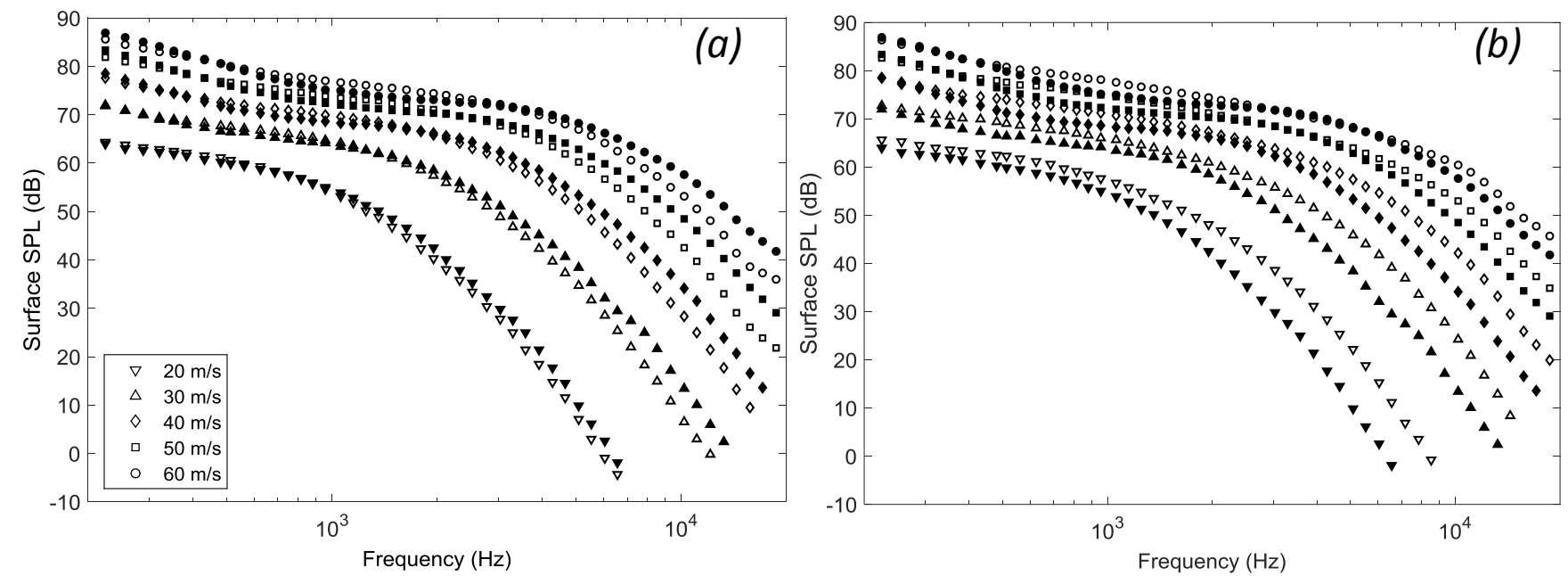

Figure 13. Surface-pressure spectra measured with the clean wall (solid symbols) and with the (a) hemispherical and (b) sandpaper rough surfaces (open symbols). Spectral levels are $1 \mathrm{~Hz}$ bandwidth SPL and are shown for different jet exit velocities $U_{j}$ 

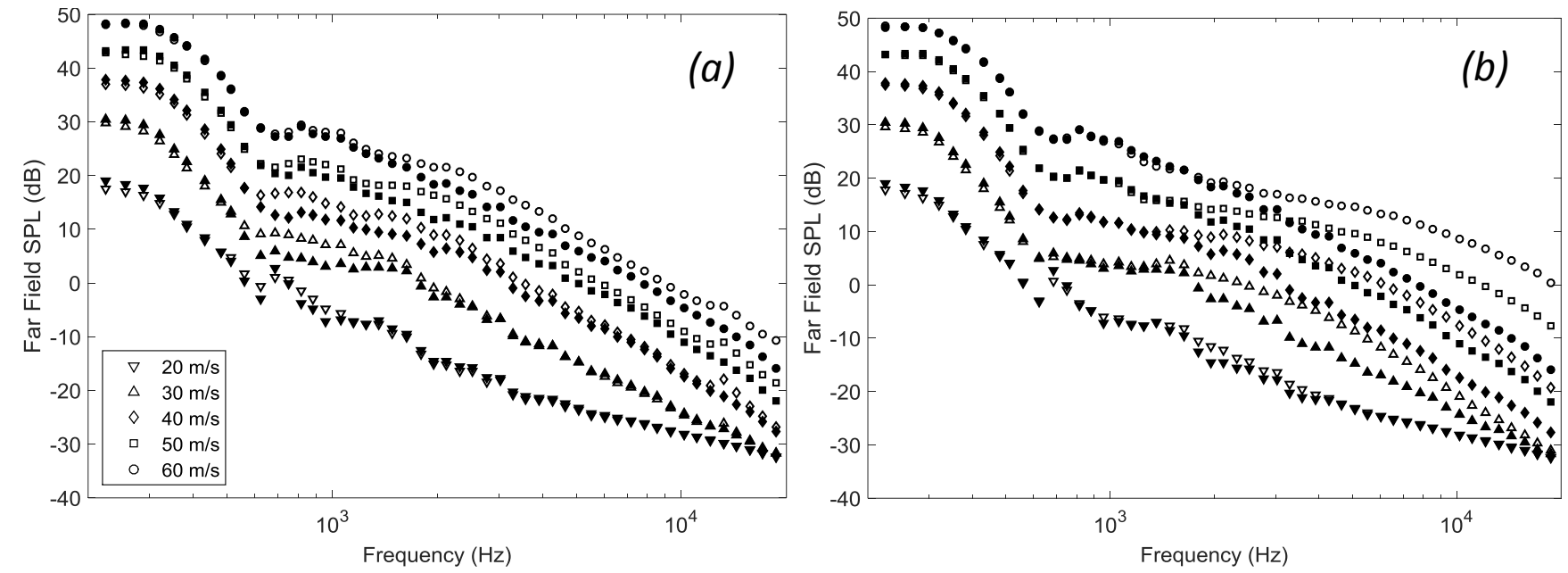

Figure 14. Background far-field noise measurements made with the clean wall (solid symbols) and with the addition of roughness noise from the (a) hemispherical and (b) sandpaper rough surfaces (open symbols). Spectral levels are $1 \mathrm{~Hz}$ bandwidth SPL and are shown for different jet exit velocities $U_{j}$ 

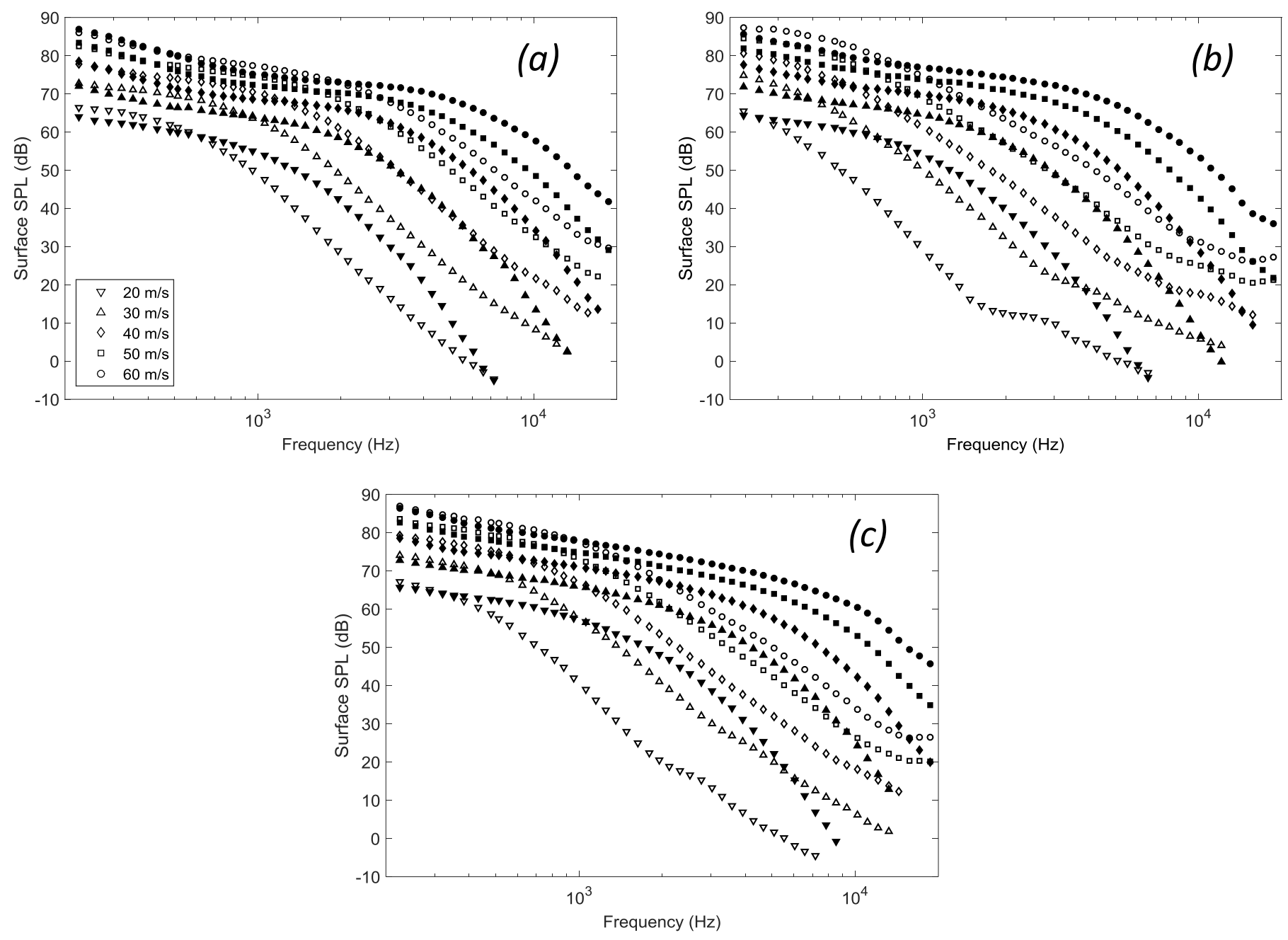

Figure 15. Surface pressure fluctuations measured with fabric canopy 5 shrouding (a) the smooth wall, and the (b) hemispherical and (c) sandpaper rough surfaces (open symbols). Solid symbols show pressure fluctuation spectra recorded for each of these surfaces, respectively, without canopy. 

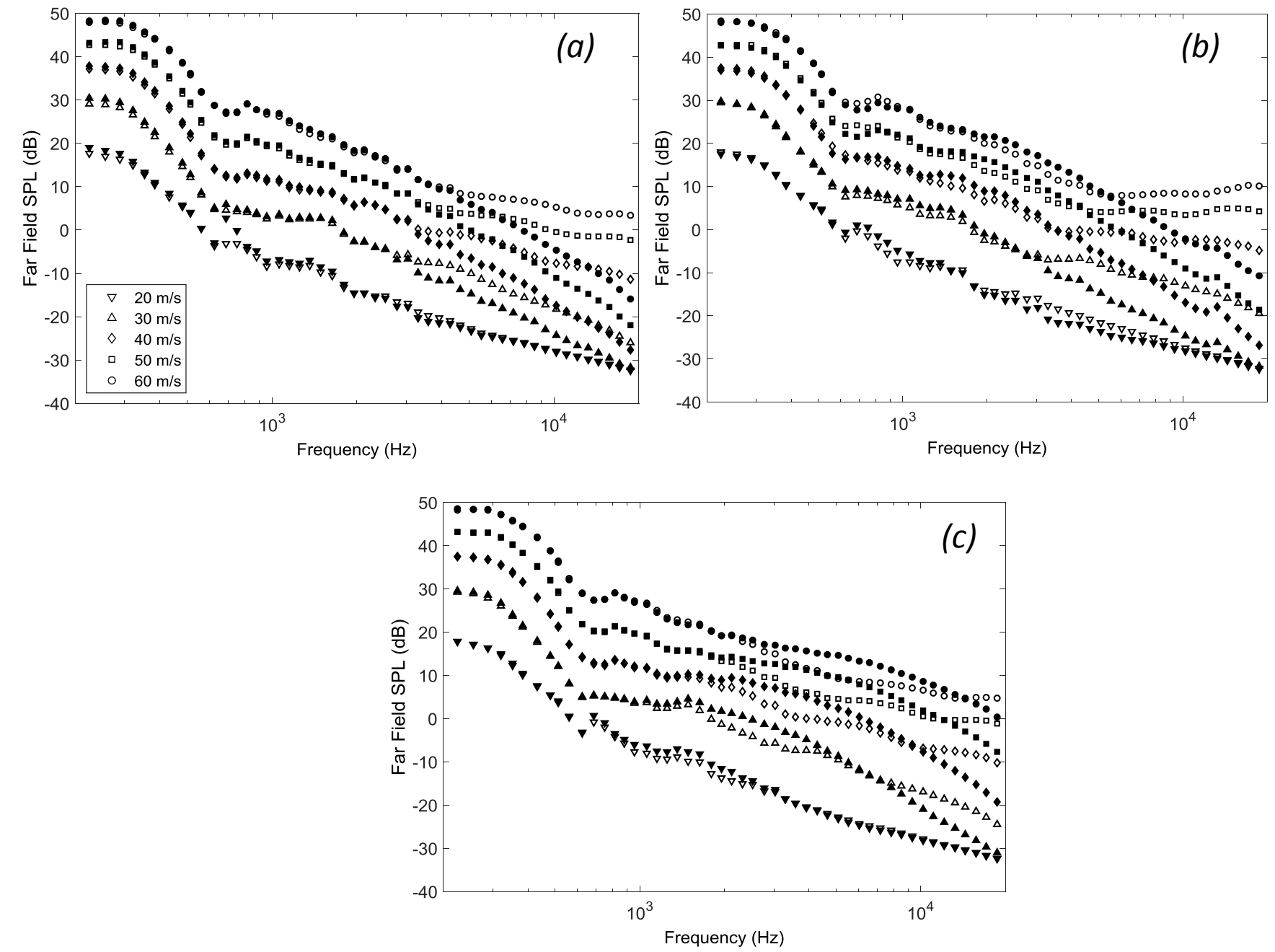

Figure 16. Noise measurements made with fabric canopy 5 shrouding (a) the smooth wall, and the (b) hemispherical and (c) sandpaper rough surfaces (open symbols). Solid symbols show sound levels recorded for each of these surfaces, respectively, without canopy. 


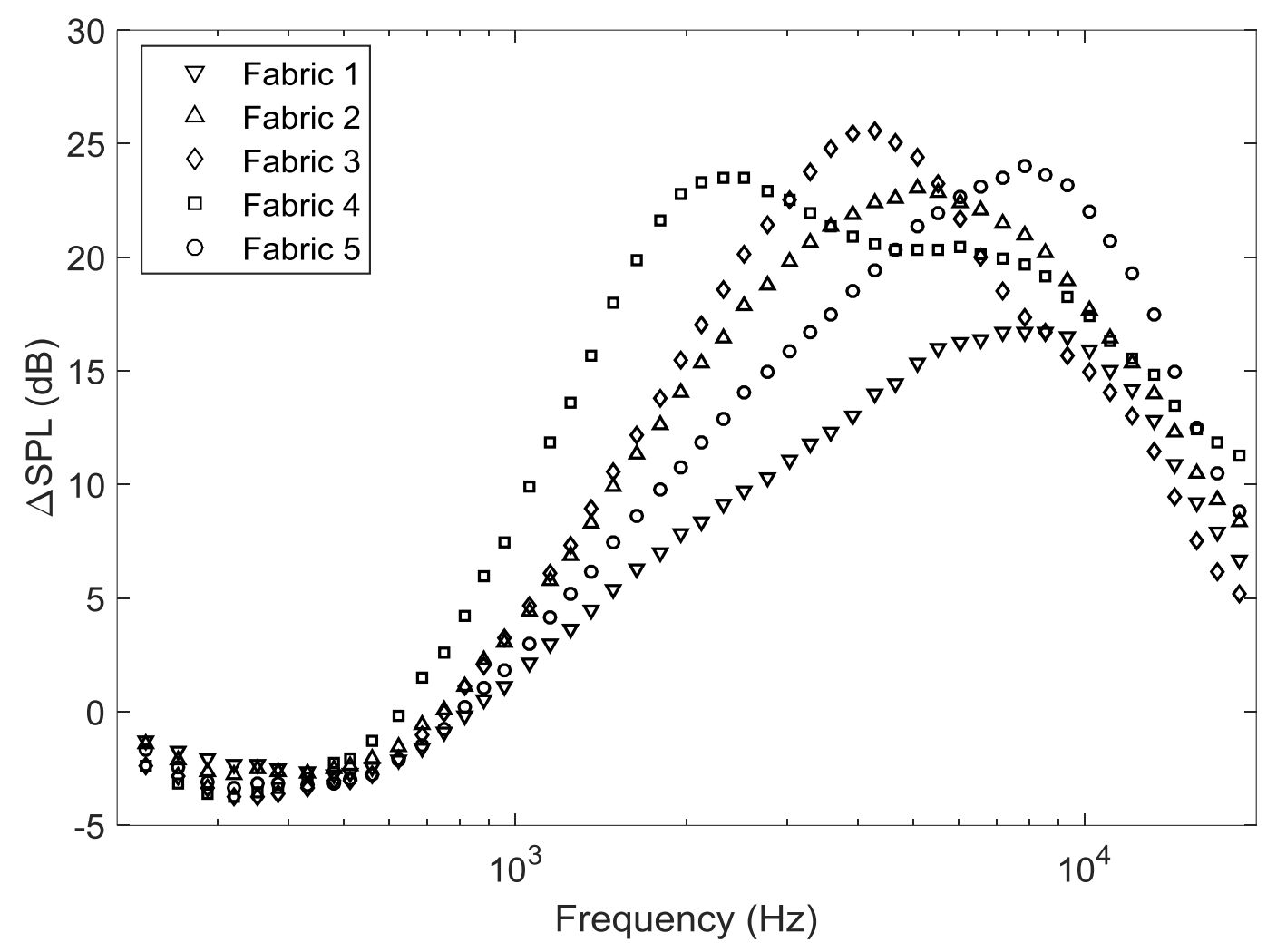

Figure 17. Attenuation of surface pressure fluctuations for each canopy over $3 \mathrm{~mm}$ roughness at $U_{j}=60 \mathrm{~m} / \mathrm{s}$. 

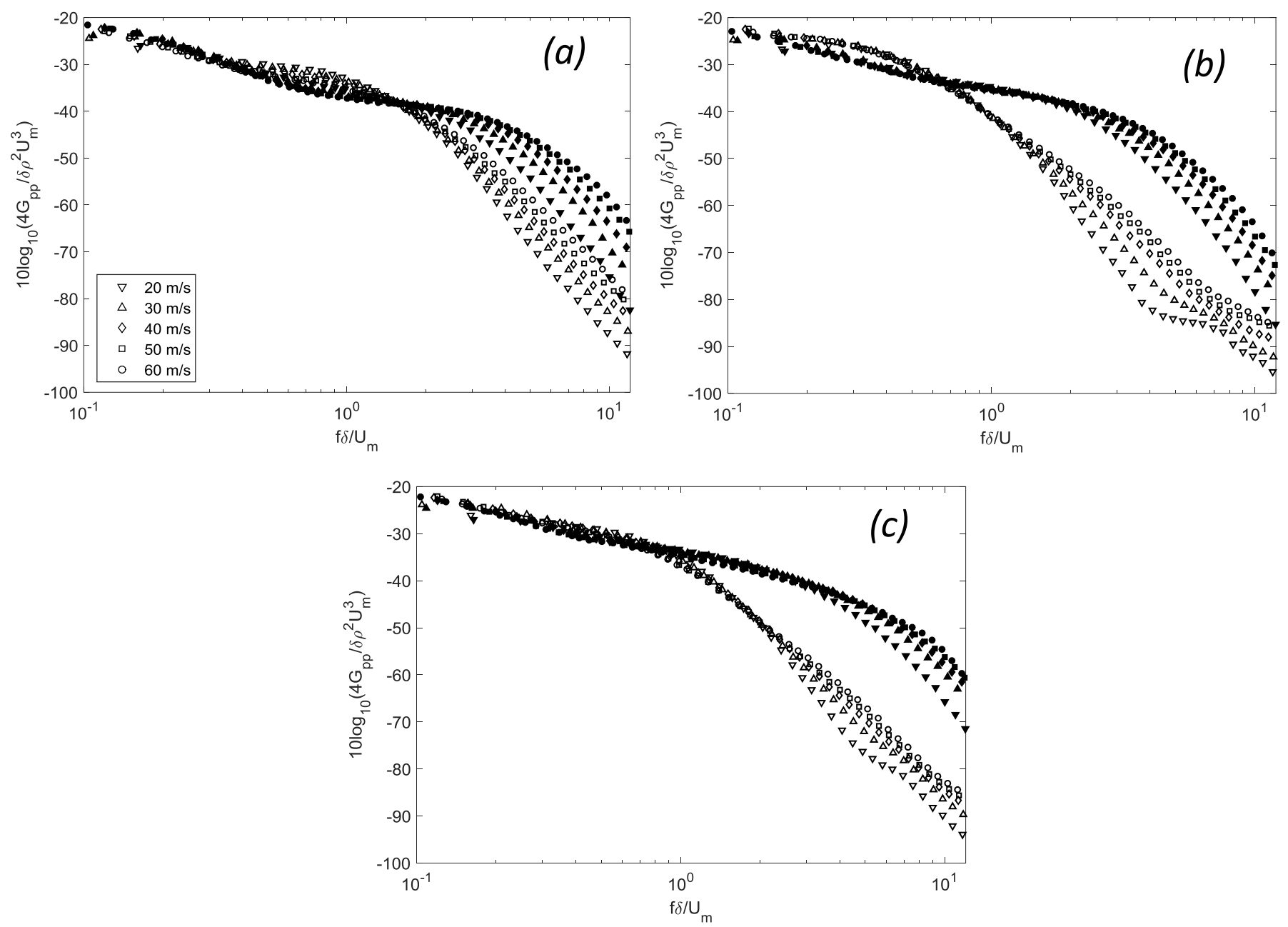

Figure 18. Scaled surface pressure fluctuations measured with fabric canopy 5 shrouding (a) the smooth wall, and the (b) hemispherical and (c) sandpaper rough surfaces (open symbols). Solid symbols show pressure fluctuation spectra recorded for each of these surfaces, respectively, without canopy. 


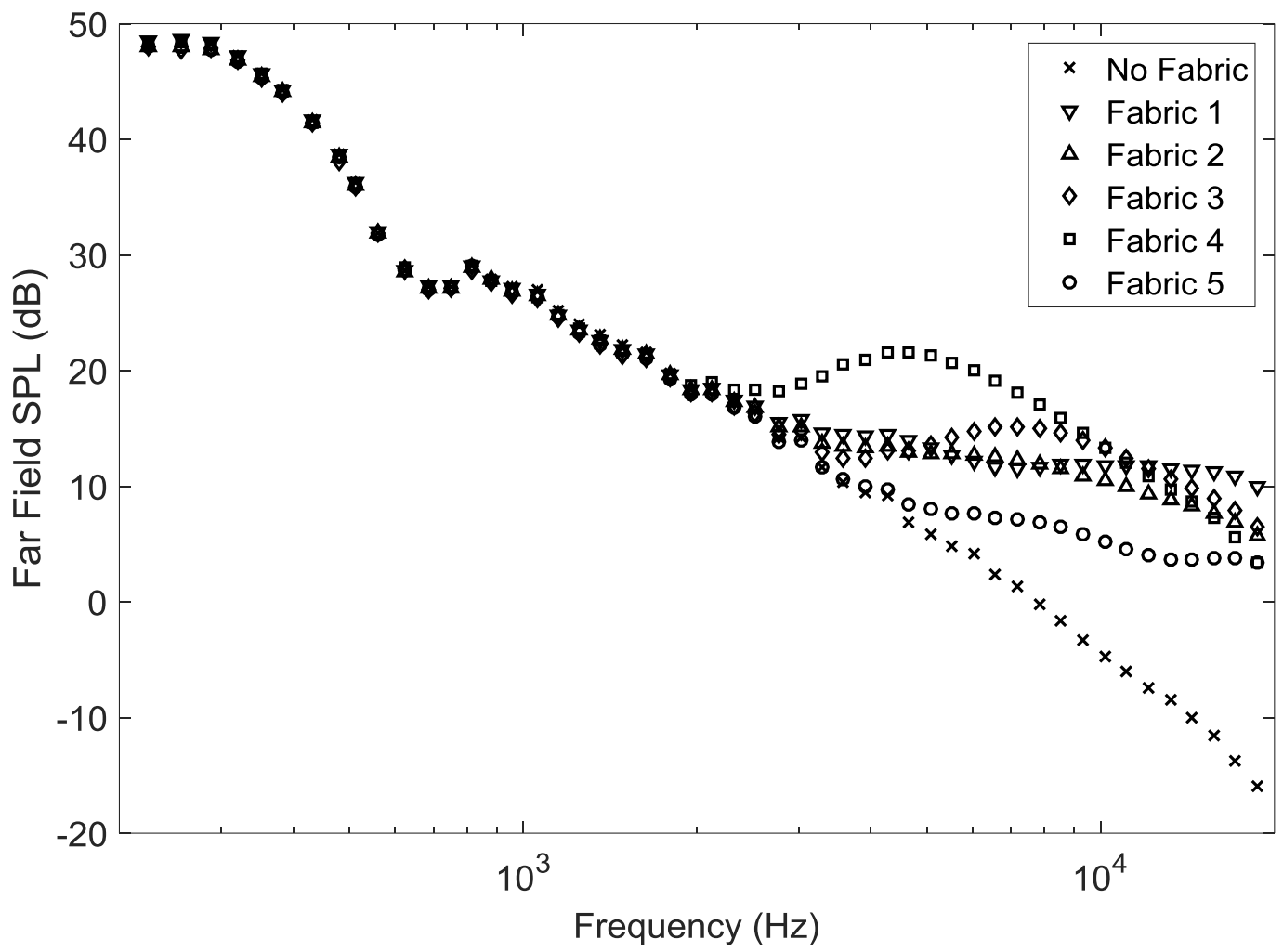

Figure 19. Comparison of far-field noise for each fabric suspended above a clean wall at $\mathrm{U}_{\mathrm{j}}=60 \mathrm{~m} / \mathrm{s}$, illustrating the self-noise of each fabric. 

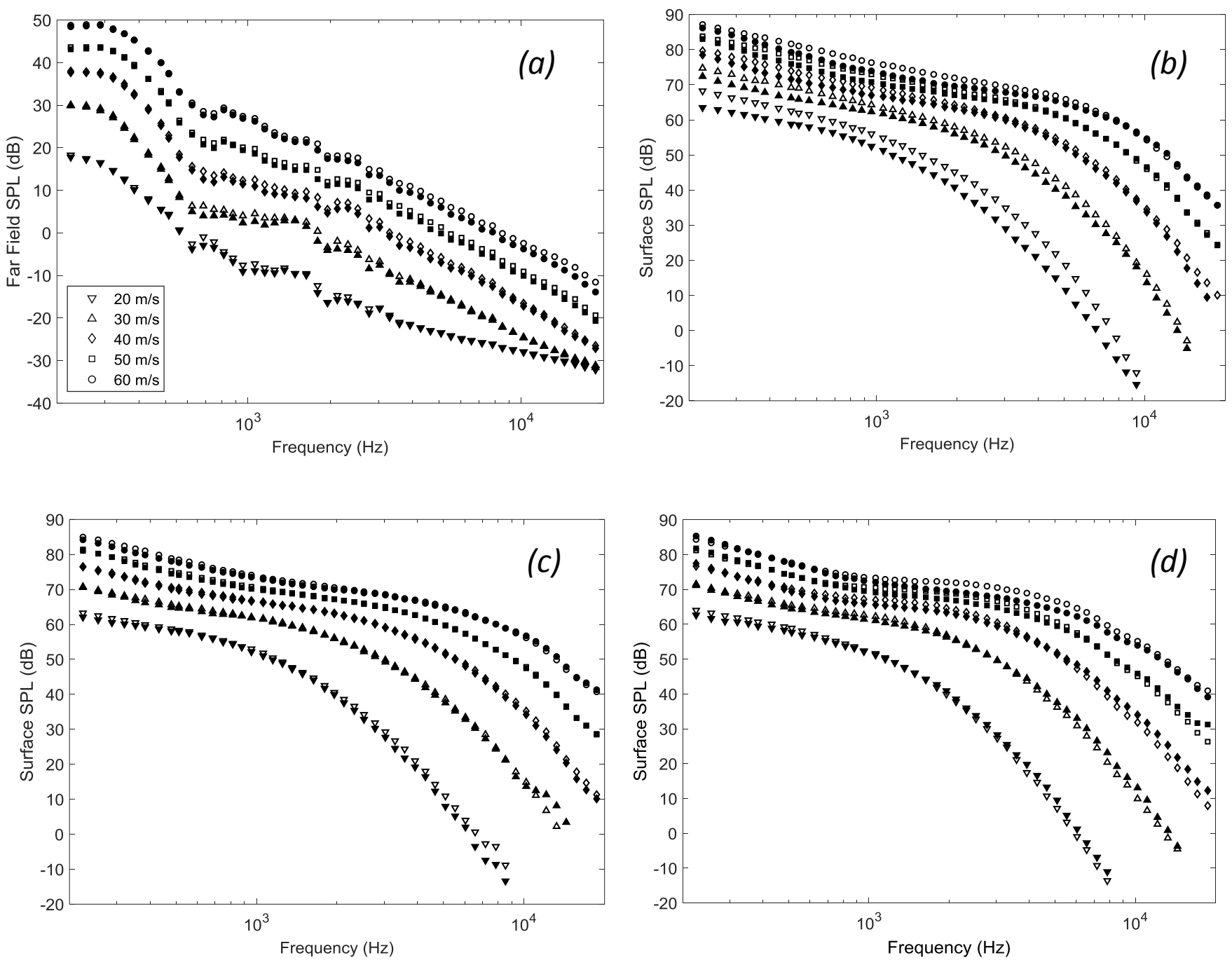

Figure 20. Measurements of (a) far-field sound and surface pressure measurements from (b) upstream, (c) middle, and (d) downstream microphones showing the effects of adding the mounts (open symbols) to the clean configuration (solid symbols). 

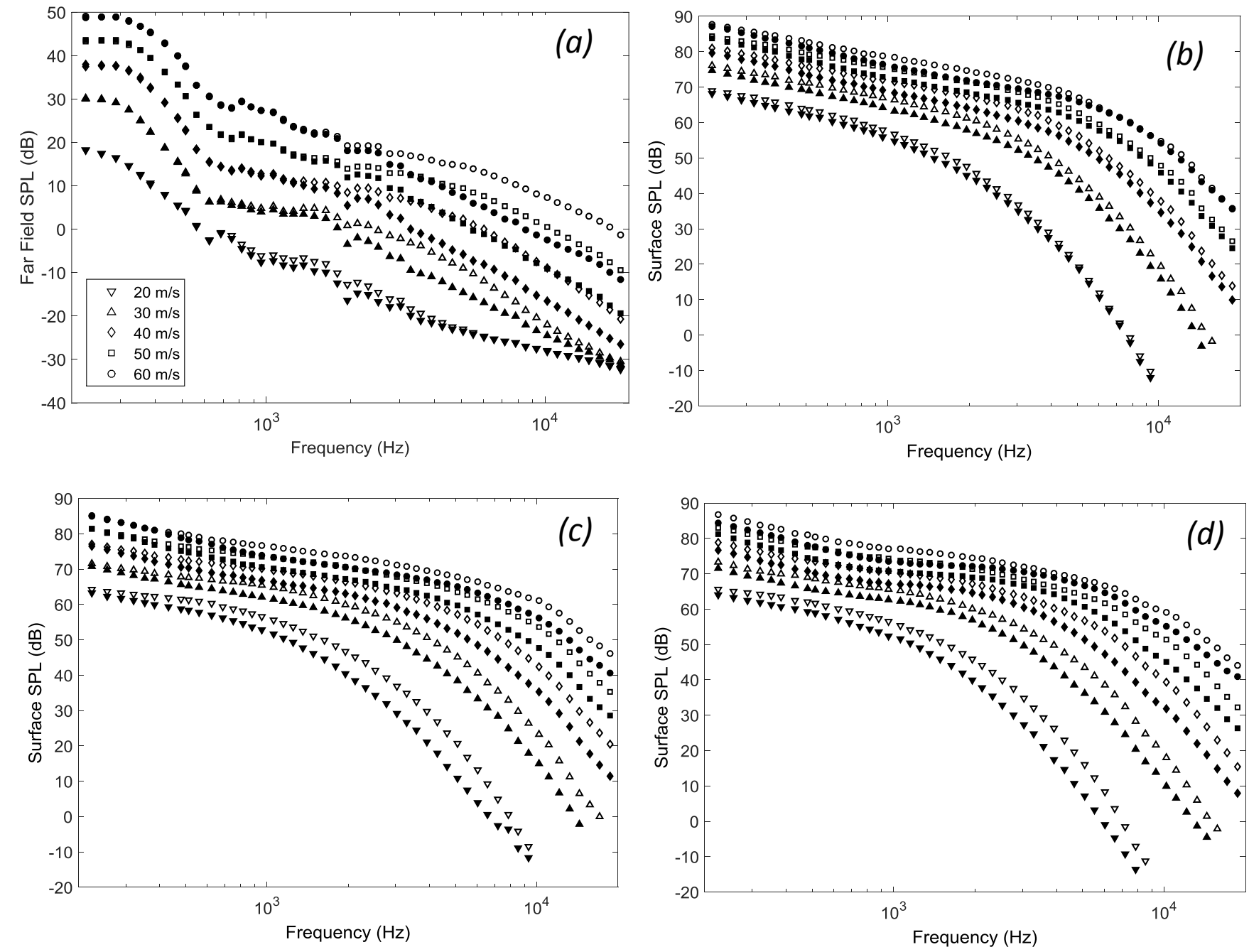

Figure 21. Measurements of (a) far-field sound and surface pressure measurements from (b) upstream, (c) middle, and (d) downstream microphones showing the effects of adding sandpaper roughness (open symbols) to the canopy mounts (solid symbols). 

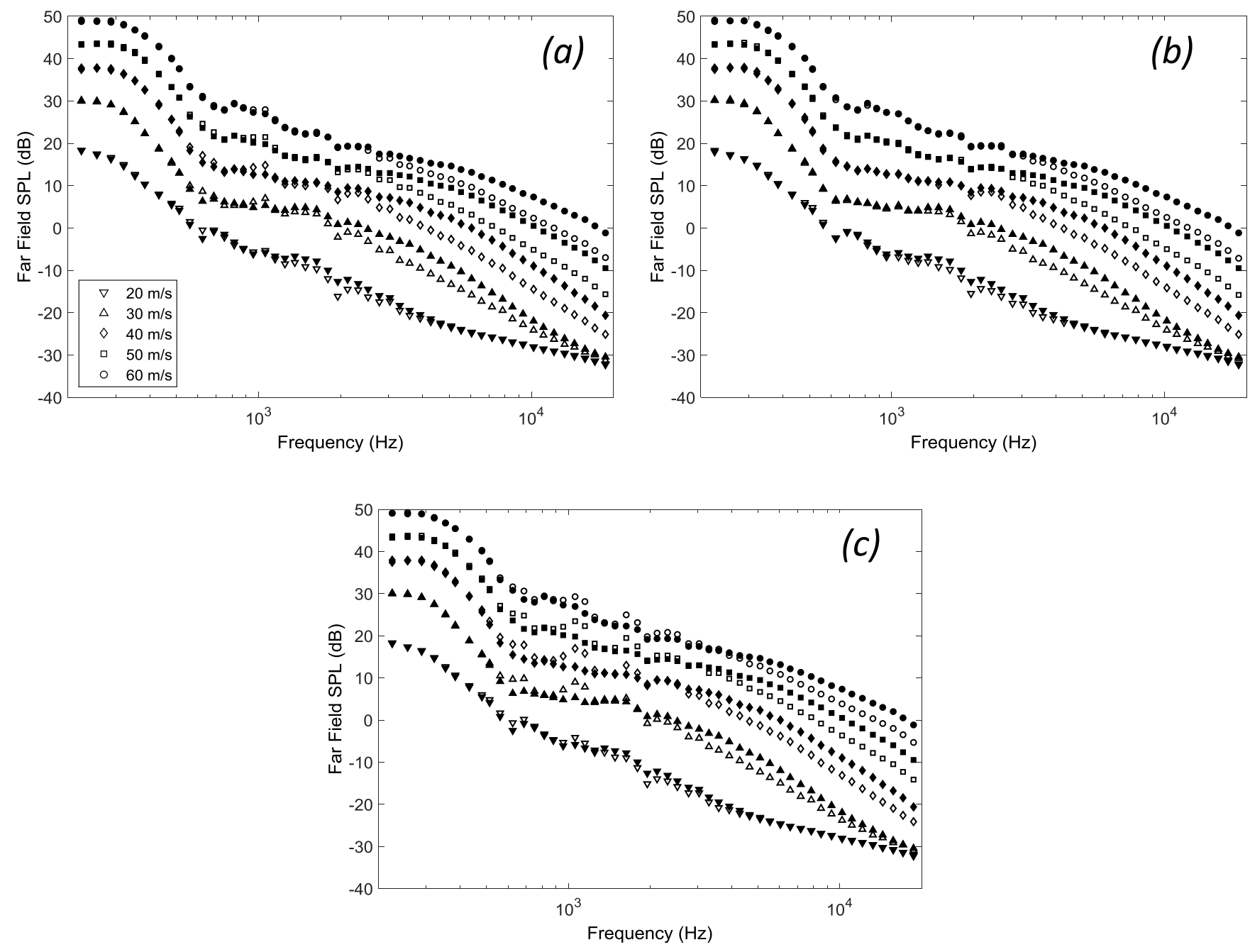

Figure 22. Measurements of far-field noise from sandpaper covered with canopies (a) 1, (b) 2, and (c) 3 (open symbols) compared with those from uncovered sandpaper (solid symbols). 

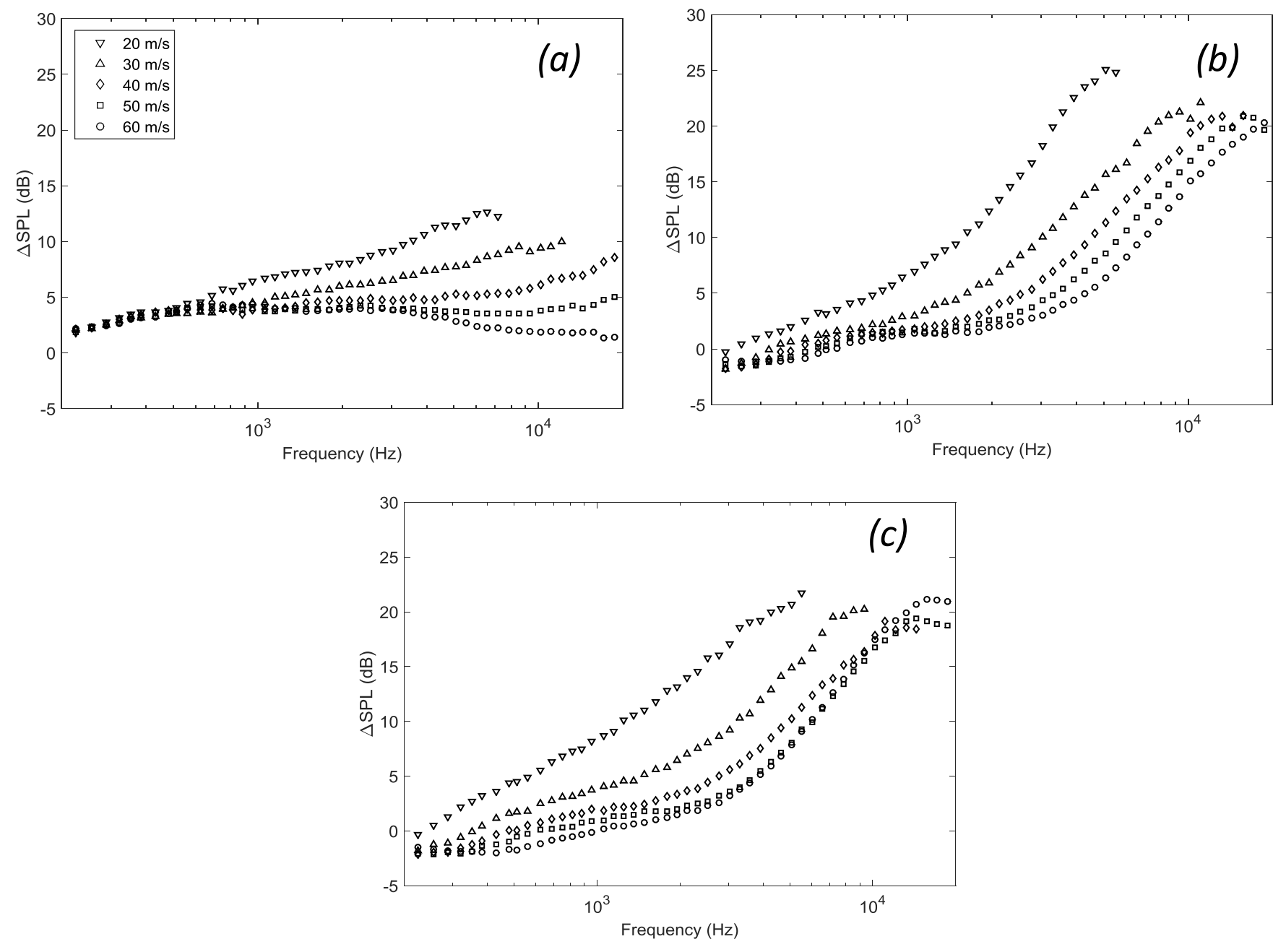

Figure 24. Surface pressure attenuation due to canopy 3 shrouding sandpaper roughness sensed by microphone (a) 1 , (b) 2, and (c) 3 . 


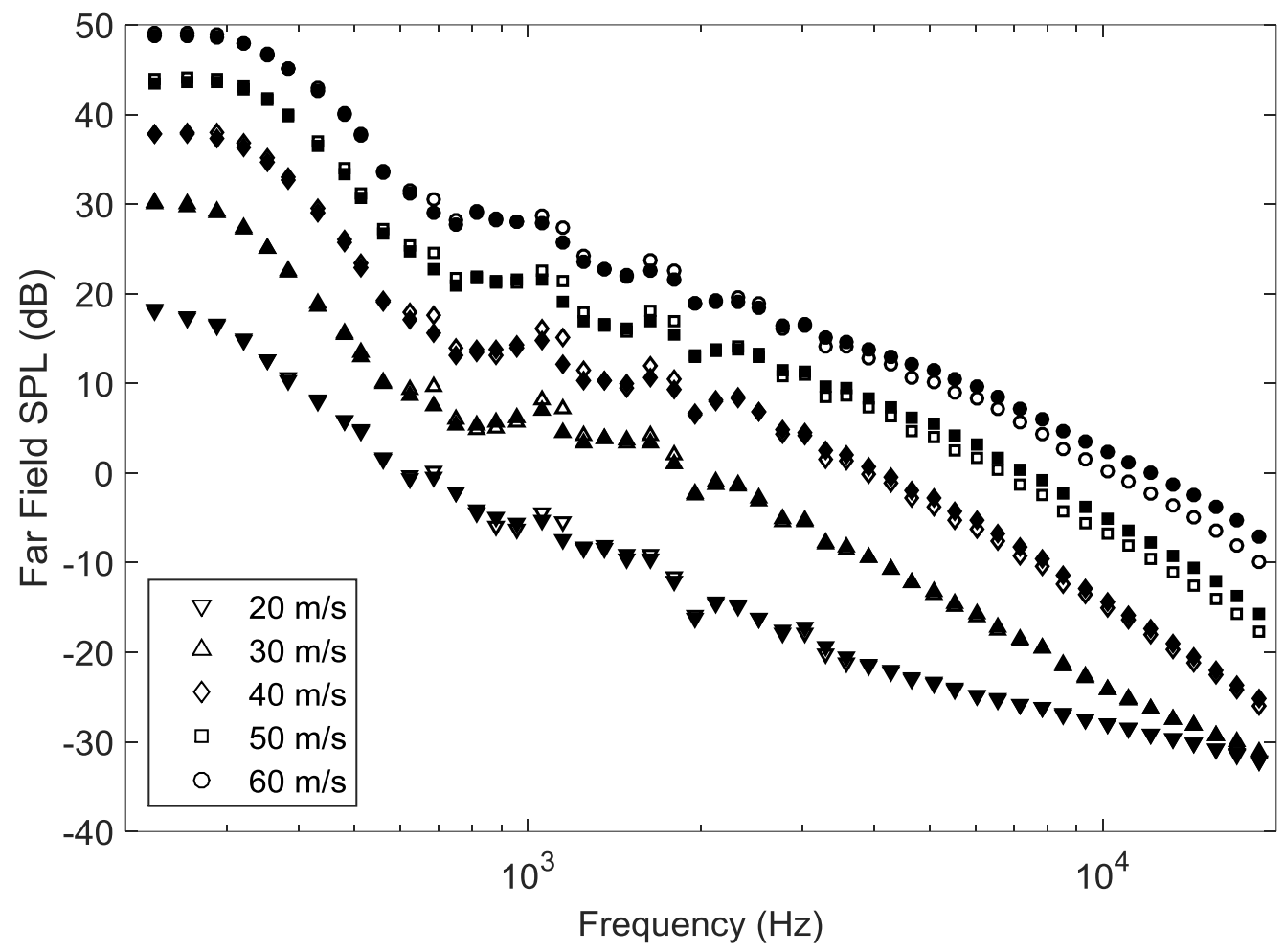

Figure 25. Measurements of far-field noise showing the difference in levels between the canopy-covered sandpaper (solid symbols) and canopy without sandpaper (open symbols). 


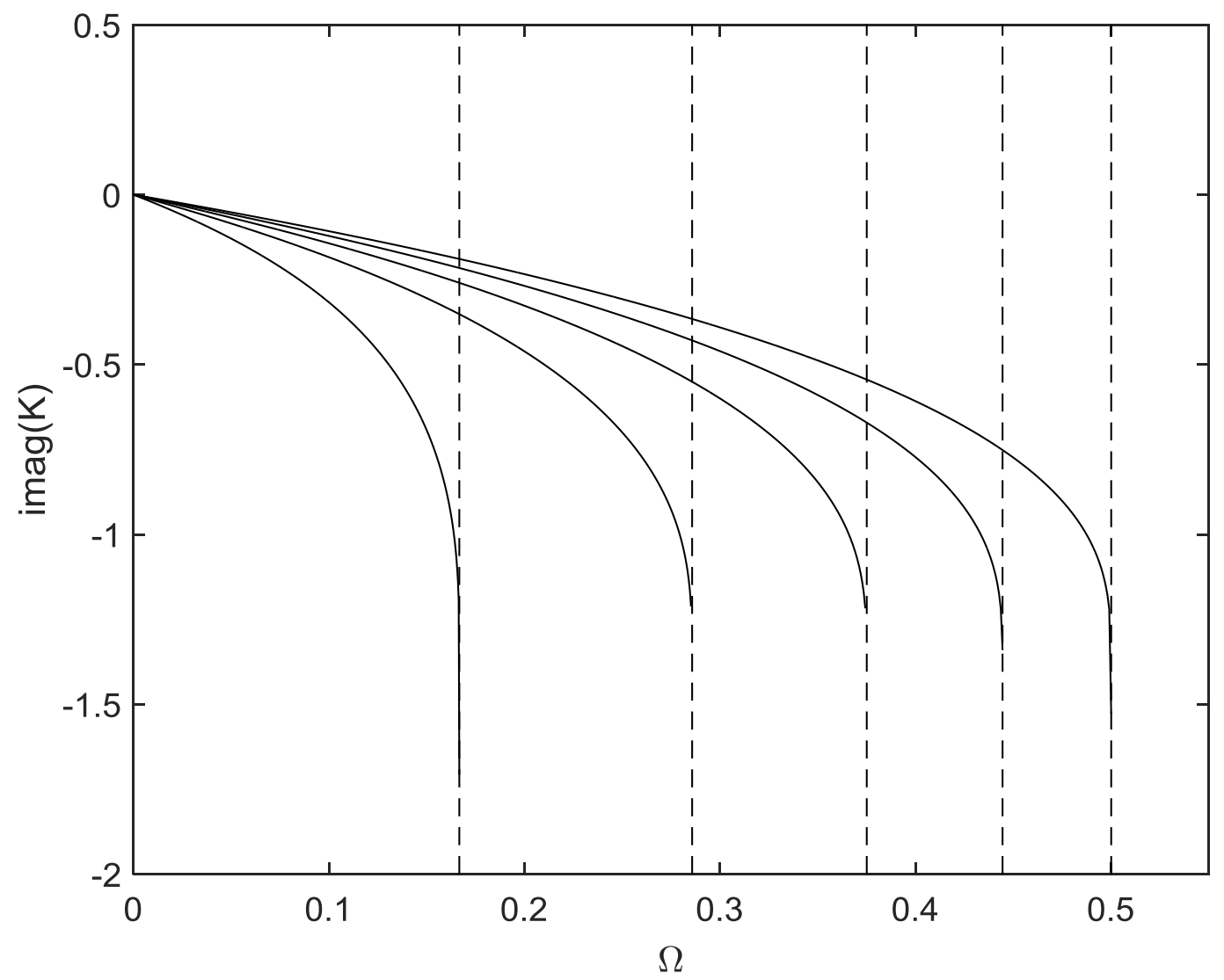

Figure 26. Mixing layer instability growth rates, for $\gamma=0.2,0.4,0.6,0.8,1$. The dashed curves are $\Omega=\gamma /(1+\gamma)$. 


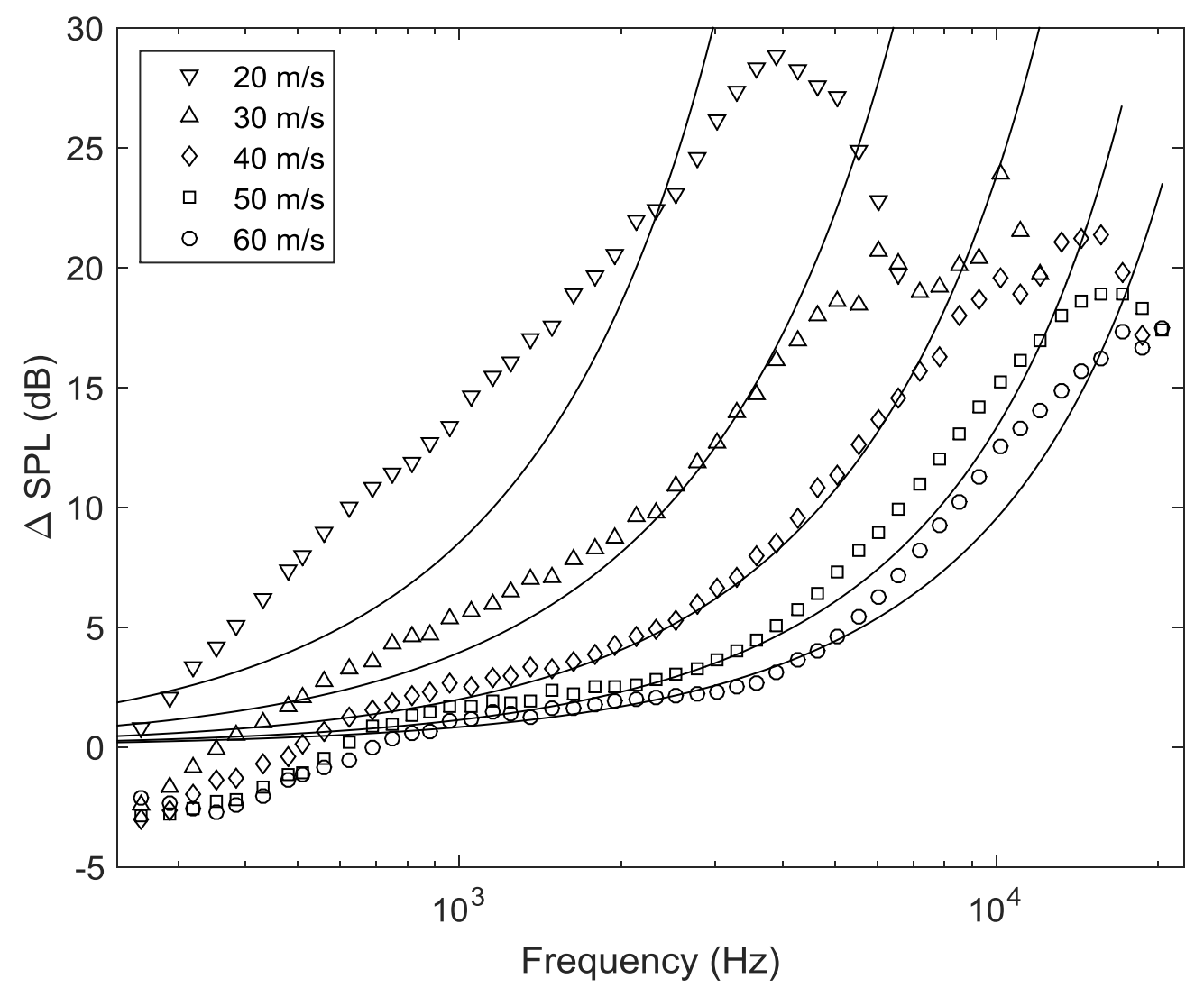

Figure 27. Comparison of experimentally measured surface pressure attenuation and theoretical prediction. 


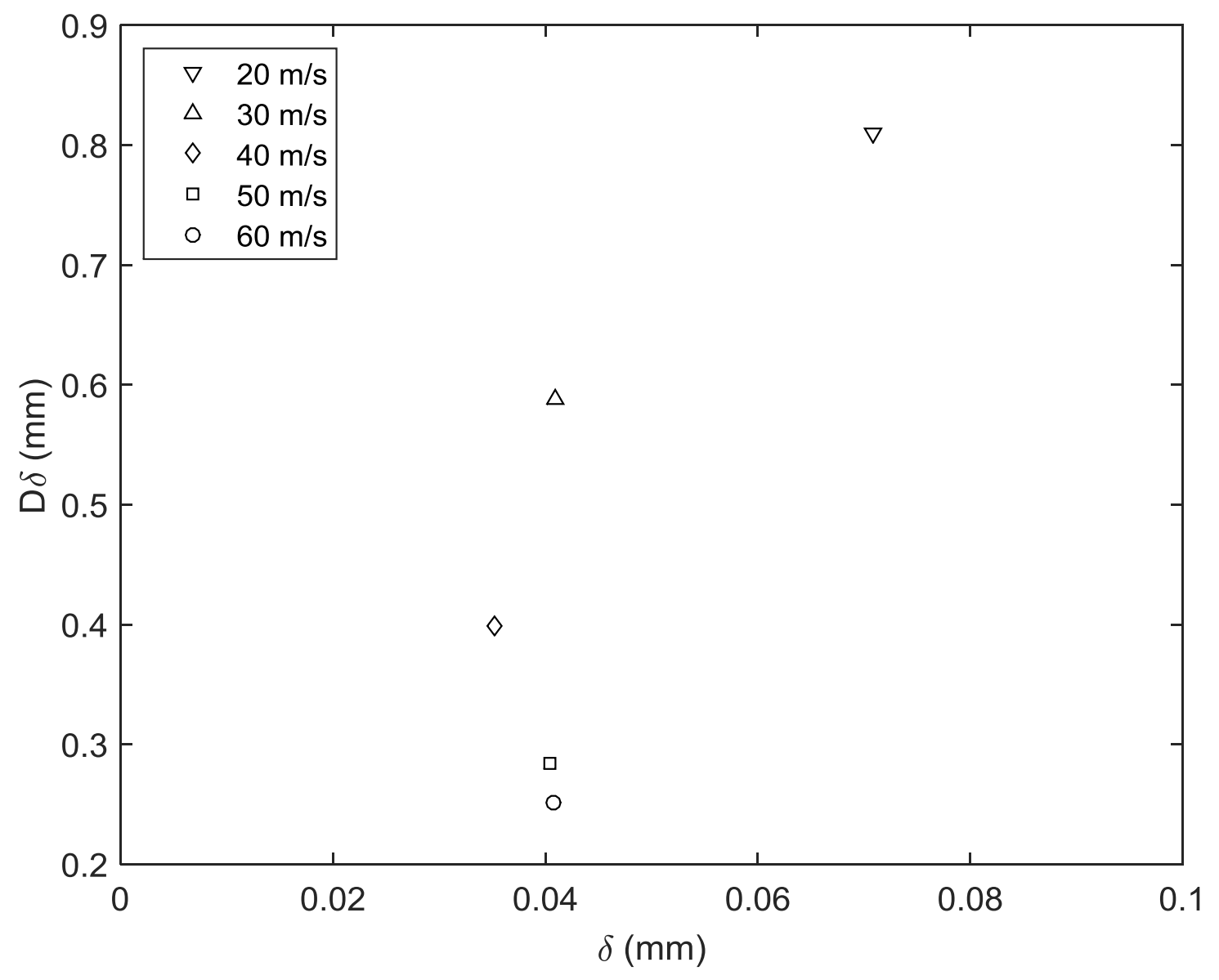

Figure 28. Plots of model parameters $\delta$ and $D \delta$ for given jet speeds. There is an approximate trend of constant layer thickness $\delta$, and decreasing length scale $D \delta$ with increasing jet velocity. 